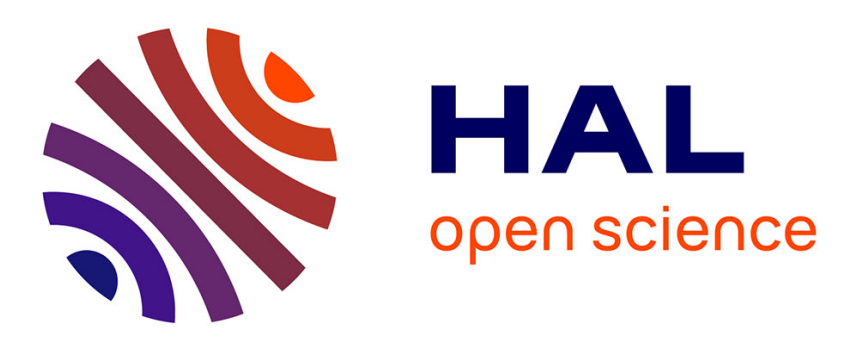

\title{
Les moules monétaires de Sées (Orne) Monnayeurs locaux et circulation du bronze au IIIe siècle apr. J.-C.
}

Pierre-Marie Guihard, François Fichet de Clairfontaine

\section{To cite this version:}

Pierre-Marie Guihard, François Fichet de Clairfontaine. Les moules monétaires de Sées (Orne) Monnayeurs locaux et circulation du bronze au IIIe siècle apr. J.-C.. Revue Numismatique, 2015, 172, pp.257-292. hal-02025098

\section{HAL Id: hal-02025098 \\ https://hal.science/hal-02025098}

Submitted on 25 Feb 2019

HAL is a multi-disciplinary open access archive for the deposit and dissemination of scientific research documents, whether they are published or not. The documents may come from teaching and research institutions in France or abroad, or from public or private research centers.
L'archive ouverte pluridisciplinaire HAL, est destinée au dépôt et à la diffusion de documents scientifiques de niveau recherche, publiés ou non, émanant des établissements d'enseignement et de recherche français ou étrangers, des laboratoires publics ou privés. 


\section{REVUE}

NUMISMATIQUE

Dirigée par

Fr. Duyrat, C. Grandjean, C. Morrisson,

M. Bompaire, A. Suspène
Secrétaires de la rédaction

V. Drost, J. Jambu, J. Olivier

2015

(172 ${ }^{\mathrm{e}}$ volume) 


\title{
Les moules monétaires de Sées (Orne)
}

\author{
Monnayeurs locaux et circulation du bronze au mie siècle apr. J.-C.
}

\begin{abstract}
Résumé - La découverte de 77 moules d'argile à Sées (Orne), lors d'opérations préventives conduites par le Service régional de l'Archéologie de Basse-Normandie entre 1992 et 1993, atteste l'existence d'une officine de faux-monnayage installée au cœur de l'antique cité des Sagiens. La marque distinctive de cette officine est la production de bronzes coulés. Le parallèle le plus direct est celui des Limesfalsa de la région du Danube. Mais à la différence des Limesfalsa, ce sont les bronzes de la période des Antonins présentant une effigie radiée qui ont été préférentiellement surmoulés par les faussaires. Les découvertes de Sées permettent de revenir sur la prime donnée à l'effigie radiée dans la circulation, révélant en contrepoint une activité frauduleuse éminemment opportuniste et liée à la politique monétaire de Postume.
\end{abstract}

Mots clés - Moules monétaires, officine, faux-monnayage, bronzes coulés, effigie radiée, Postume.

Summary - 77 clay moulds were discovered in Sées (Orne) during preventive operations carried over by the Service régional de l'Archéologie de Basse-Normandie in 1992-1993. They document the existence of a counterfeiters' workshop located in the center of the ancient city of the Sagii. It produced cast bronze coins. The closest parallel is that of the Danubian Limes falsa. The counterfeiters preferred cast coins were the Antonines' radiates. This discovery shows the premium given to radiate types in the circulation, closely related to Postumus monetary policy.

Keywords - Coin moulds, workshop, counterfeiting, cast bronze coins, radiate figures, Postumus.

Dans l'histoire romaine, avec les frappes officielles, la fausse monnaie et l'imitation sont des éléments inséparables de la circulation monétaire. On observe ainsi tout au long de l'Empire des vagues épisodiques de productions monétaires, qui usurpent en quelque sorte la détention du ius monetae en reproduisant par différents procédés des émissions officielles. De nombreux numismates qualifient ce phénomène d' "épidémies», puisqu'il semble toujours se limiter à une région et à un laps de temps déterminé ${ }^{1}$.

Les découvertes de moules de terre cuite, portant l'empreinte de bronzes du Haut-Empire, à Sées (Orne) prennent précisément place dans le débat sur les productions monétaires non officielles. Surtout, elles ont l'avantage d'avoir été pour partie documentées par un contexte archéologique suggérant l'existence

* Université de Caen Basse-Normandie, Ingénieur-docteur, responsable du service de numismatique du Centre Michel de Boüard-CRAHAM, UMR 6273.

Courriel : pierre-marie.guihard@unicaen.fr.

** Inspecteur général des patrimoines (Ministère de la Culture et de la Communication). Courriel : francois.fichet-de-clairfontaine@culture.gouv.fr.

1. King 1996, p. 237. Terminologie reprise par Peter 2004, p. 26-29. 
d'activités artisanales au sein de quartiers urbains situés en périphérie de la capitale antique de la cité des Sagiens. Elles offrent la possibilité, non des moindres, de replacer une pratique dans un contexte défini. Par conséquent, la présente enquête ne portera pas seulement sur les moules eux-mêmes, mais autant sur l'environnement de la découverte et le cadre historique et économique qui a vu la pratique se développer. Il sera alors possible de réfléchir sur les buts et les moyens d'une activité de surmoulage à l'ombre des murs de la cité sagienne.

\section{Présentation et intérêt de la découverte}

L'ensemble étudié compte 80 moules et fragments mis au jour à deux endroits différents de la ville ${ }^{2}$, le quartier antique de la rue Amesland et le site de l'église de l'Immaculée Conception ${ }^{3}$. Ces valves d'argile, destinées à la fabrication de monnaies par surmoulage, portent toutes une empreinte de droit et de revers. Leur identification dénote d'emblée la prédominance de types monétaires ancrés dans le Haut-Empire (tableau 1). La reproduction d'émissions du Ier siècle y est rare. En revanche, celles du IIe siècle abondent. L'examen des droits est en cela significatif : 1 moule se rattache à la période flavienne tandis que 24 autres renvoient à des émissions antonines où dominent de façon équilibrée les effigies de Trajan (8), Hadrien (9) et Antonin le Pieux (7). Un même constat s'impose fort logiquement pour les empreintes de revers. Toutefois, la prévalence des types monétaires du IIe siècle est encore accentuée par l'absence totale de représentant du jer siècle.

On s'étonnera tout autant du resserrement des dénominations. C'est ce que suggèrent très clairement les monnaies utilisées pour la prise d'empreintes (tableau 1) : il s'agit sans exception de bronzes, des dupondii et des as qui ont pour dénominateur commun la présentation quasi systématique au droit d'une tête radiée 4 . Sur 39 empreintes de droit, 24 têtes radiées et 2 têtes laurées ont été comptabilisées, les 13 empreintes restantes étant indéterminées ou illisibles.

2. Fichet DE Clairfontaine 1998, p. 19-43.

3. Les découvertes de la rue Amesland comptent aujourd'hui parmi les collections du musée de Normandie. Nous remercions J.-M. Lévesque (directeur), S. Berthelot (conservatrice) et L. Voracek (attachée de conservation) d'avoir mis à notre disposition les moules le temps de leur étude. Quelques moules mis au jour sur le site du Petit Séminaire ont été retrouvés aux Archives départementales de l'Orne. Nous remercions J.-P. Foucher (directeur) d'avoir rendu possible leur consultation.

4. Bien que présentant une tête radiée, les empreintes à la légende de revers S C (DAC PARTHICO P M TR POT XX COS VI P P), qui renvoient à une émission «orientale» datée du règne de Trajan, sont rangées parmi les as : ce classement est précisément suivi par les auteurs du RIC II, p. 290, no 644. L'attribution et l'aire de diffusion de ces as dits «orientaux» ont été discutées par Besombes 2008, p. 27 qui prend position pour une émission frappée dans l'atelier de Rome. Cf. aussi sur le sujet l'étude de Woytek 2010. 


\begin{tabular}{|c|c|c|c|}
\hline \multicolumn{2}{|r|}{ Empreintes de droit } & $\mathrm{Nbr}$ & $\%$ \\
\hline \multirow[t]{2}{*}{ Flaviens } & & 1 & 2,50 \\
\hline & Domitien & 1 & \\
\hline \multirow[t]{4}{*}{ Antonins } & & 24 & 60,00 \\
\hline & Trajan & 8 & \\
\hline & Hadrien & 9 & \\
\hline & Antonin le Pieux & 7 & \\
\hline Dynastie antonine & & 15 & $\mathbf{3 7 , 5 0}$ \\
\hline 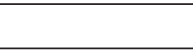 & Total & 40 & \\
\hline
\end{tabular}

\begin{tabular}{|c|c|c|c|c|}
\hline \multicolumn{2}{|r|}{ Empreintes de revers } & \multirow[b]{2}{*}{ Rue Amesland } & \multirow[b]{2}{*}{ Petit Séminaire } & \multirow[b]{2}{*}{$\%$} \\
\hline & & & & \\
\hline \multirow[t]{2}{*}{ Antonins } & Trajan & 7 & & $\mathbf{1 7 , 5 0}$ \\
\hline & DAC PARTHICO P M TR POT XX COS VI P P & 7 & & \\
\hline \multirow[t]{2}{*}{ Antonins } & Hadrien & 8 & & 20,00 \\
\hline & $\begin{array}{l}\text { FELICITATI AVG // COS III P P } \\
\text { La Fortune assise, tenant un gouvernail } \\
\text { de la main dr. et une corne d'abondance. }\end{array}$ & 5 & & \\
\hline \multirow[t]{2}{*}{ Antonins } & Antonin le Pieux & 8 & 2 & 25,00 \\
\hline & $\begin{array}{l}\text { TR POT XV COS IIII // ANNONA AVG } \\
\text { BRITAN-NIA COS IIII } \\
\text { AETERNITAS }\end{array}$ & $\begin{array}{l}7 \\
1\end{array}$ & 2 & \\
\hline \multirow[t]{2}{*}{ Dynastie antonine } & & 14 & 1 & $\mathbf{3 7 , 5 0}$ \\
\hline & $\begin{array}{l}\text { L'Espérance tenant une fleur } \\
\text { et relevant le bas de sa robe. } \\
\text { La Fortune tenant un gouvernail } \\
\text { et une corne d'abondance. } \\
\text { La Fidélité tenant des épis de blé orientés vers le bas } \\
\text { et un plateau de fruits. } \\
\text { Junon assise, tenant une patère } \\
\text { Personnage debout, tenant une corne d'abondance } \\
\text { Type indéterminé }\end{array}$ & $\begin{array}{l}1 \\
1 \\
3 \\
1 \\
1 \\
8\end{array}$ & 1 & \\
\hline & Total & 37 & 3 & 100,00 \\
\hline
\end{tabular}

\begin{tabular}{|l|l|c|c|}
\hline \multicolumn{1}{|c|}{ Dénomination } & Nbr & \% \\
\hline & As & 13 & 16,25 \\
& Dupondius & 41 & 51,25 \\
& As ou Dupondius & 26 & 32,50 \\
\hline \multicolumn{2}{r|}{ Total } & $\mathbf{8 0}$ & \\
\hline
\end{tabular}

Tableau 1 - Présentation des moules et fragments découverts à Sées (Rue Amesland et Petit Séminaire, église de l'Immaculée Conception). 
Aussi doit-on conclure que le surmoulage d'espèces radiées émises sous la Dynastie des Antonins a été ici privilégié.

Portant tous l'empreinte de monnaies de bronze du Haut-Empire, les moules monétaires de Sées présentent d'ores et déjà une identité particulière qui les distingue d'autres découvertes ${ }^{5}$. Précisément, les activités de coulage de monnaies officielles identifiées en Gaule et en Britannia ${ }^{6}$ visaient surtout la reproduction de deniers et d'antoniniens des IIe-IIIe siècles. Le surmoulage de monnaies de bronze semble être une pratique géographiquement peu répandue ${ }^{7}$. On trouve ainsi une production de bronzes modelés sur les territoires du haut et moyen Danube. Ce sont ce que les numismates appellent des Limesfalsa, attestés en masse dans les trouvailles de ces régions ${ }^{8}$. Des moules de bronzes ont été également enregistrés en Britannia (Londres, etc.) et en Gaule Belgique (Saint-Mard, Pachten, etc.), où ils sont complémentaires d'une production de faux deniers. Toutefois, cette fabrication n'a été nulle part aussi intense que sur les territoires danubiens ${ }^{9}$, ne rendant que plus originales les découvertes de Sées. Il apparaitrait, dès lors, qu'il y avait au cour des échanges de la cité des Sagiens un réel intérêt à reproduire exclusivement des monnaies de bronzes et de surcroît à l'effigie radiée.

\section{Les moules monétaires de Sées}

\section{Les moules de la rue Amesland}

L'essentiel des moules monétaires (>95\%) provient d'un secteur situé en bordure septentrionale de l'agglomération gallo-romaine de Sées, au niveau de l'actuelle rue Amesland (figure 1). Ce secteur a été exploré à l'occasion de deux opérations préventives conduites par le Service régional de l'archéologie de BasseNormandie entre 1992 et 1993. Le mobilier recueilli et les structures identifiées ont révélé une occupation dense des lieux à partir de la première moitié du Ier siècle et à forte vocation artisanale au cours des IIe et IIII siècles (figure 2$)^{10}$.

5. Sur le phénomène plus général des productions monétaires non officielles voir le bilan dressé par PETER 2004.

6. Un premier inventaire avait été dressé en 1994 par J. Lallemand. En 2003, G. Aubin le met à jour et recense 88 lieux de découverte de moules monétaires du IIIe siècle. Pour la bibliographie correspondante : LALLEMAND 1994, p. 141-177 ; Aubin 2003, p. 125-162.

7. Voir l'utile tableau de synthèse publié dans Chameroy 2007,p. 542-543. Dans cette étude, l'auteur dresse la composition de toutes les découvertes de moules monétaires actuellement connues.

8. Gazdac, Alföldy-Gazdac 2001, p. 137-145 ; Vondrovec 2003 ; Pfisterer 2007, p. 648 736. Quelques découvertes de monnaies de bronze coulées du Haut-Empire ont été également signalées en Gaule : voir notamment BASTIEN 1970, p. 475-477.

9. Supra n. 8. Voir aussi Kunisz 1980, p. 135-136. Étude qui pourra être complétée par Boon 1965, p. 161-174; Callu 1969, p. 125, n. 1.

10. Mandy 1992, p. 55 ; Fichet de Clairfontaine 1998 ; Bernouis 1999, p. 199. 


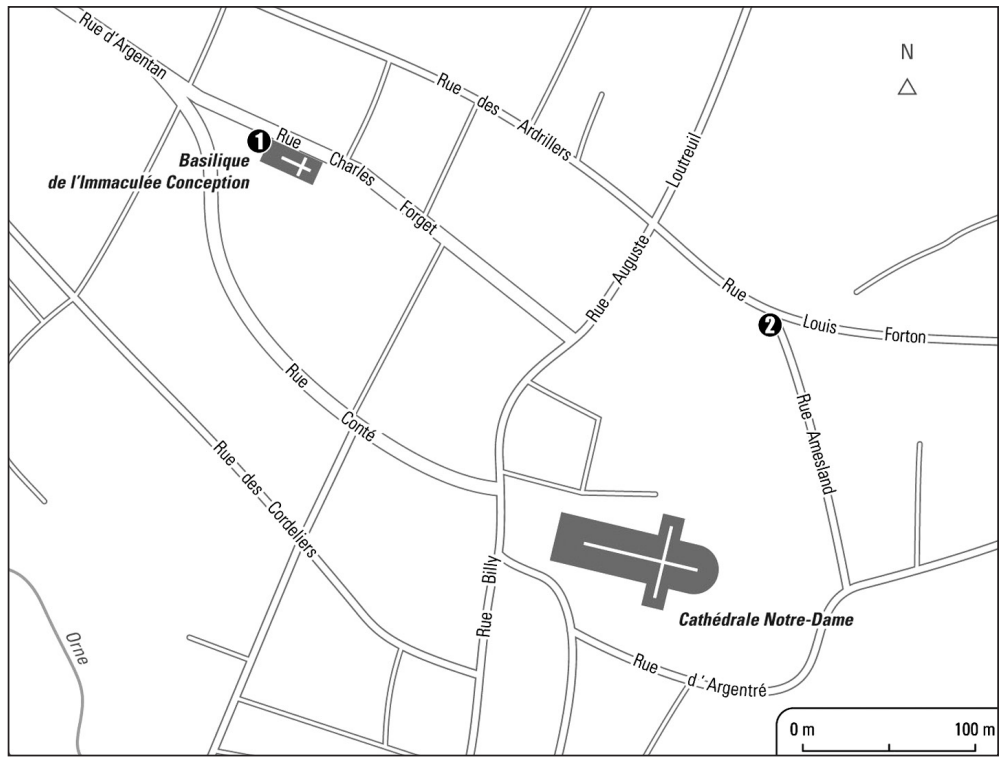

Figure 1 - Localisation des découvertes de moules monétaires à Sées $(1:$ Petit Séminaire, église de l'Immaculée Conception, vers 1855 ; 2 : Rue Amesland, 1992-1993) (DAO : Jean-Claude Fossey, UMR 6273, CRAHAM).

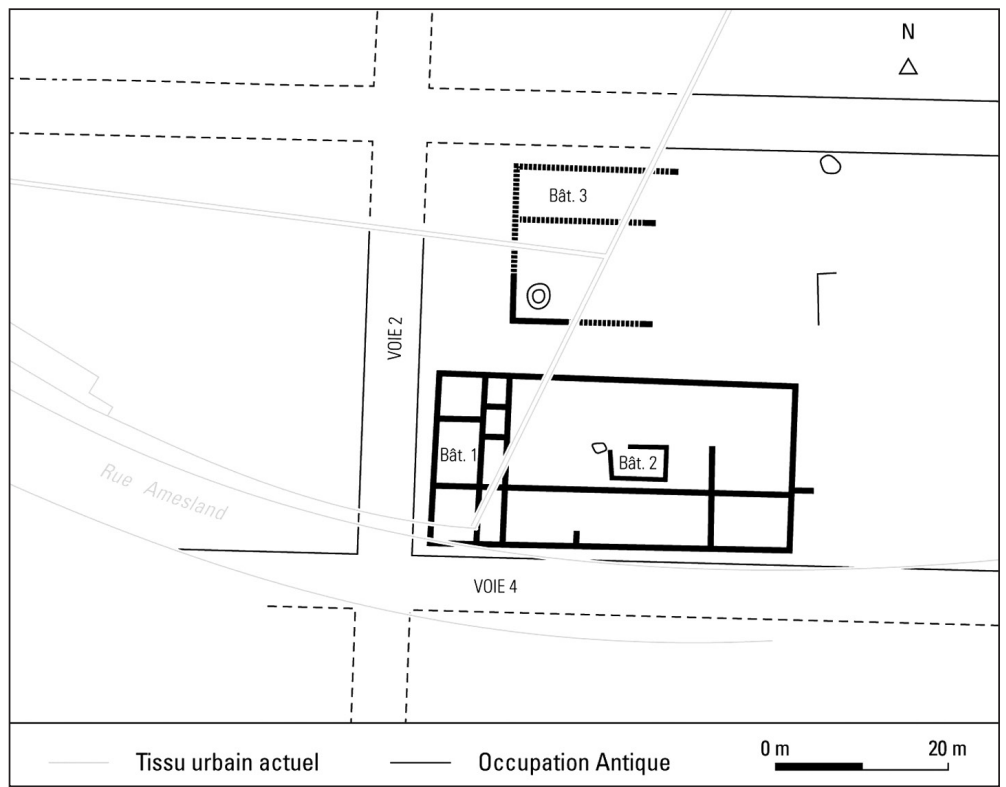

Figure 2 - Sées, rue Amesland. Plan des vestiges gallo-romains (reprise DAO : Jean-Claude Fossey, UMR 6273, CRAHAM). 
La première fouille conduite en 1992 le long de la rue Amesland, qui emprunte pour partie le tracé d'une voie antique, a livré les vestiges d'une première occupation marquée par des architectures de terre et de bois. Elle a été remplacée à partir de la seconde moitié du ier siècle par des constructions maçonnées proches de l'angle sud-ouest d'un quartier urbain. L'hypothèse privilégiant un vaste édifice cloisonné par des boutiques en rez-de-chaussée peut être avancée. Toutefois, l'ensemble associé à des foyers, des petits fours à chambre unique et des niveaux de rejets cendreux a été fortement arasé lors de son abandon dans la seconde moitié du III siècle, peut-être durant le dernier tiers. La plus importante série de moules en terre a été recueillie au sein du comblement d'un fossé ou d'une tranchée qui a recoupé la voie antique à une période indéterminée mais qui semble bien antérieure au Ive siècle. En 1993, une fouille conduite dans une parcelle adjacente a livré l'angle sud-ouest du quartier urbain. C'est à l'emplacement du grand édifice qu'une construction s'est élevée au cours du IIIe siècle, abritant un four ainsi qu'un grand puits quadrangulaire maçonné comblé de matériel du IIIe siècle et qui contenait deux moules en terre cuite et des fragments de creusets. Tout proche à l'extérieur, subsistaient des fours à soles en terre cuite associés à des rejets de bronze. Le matériel céramique recueilli dans le comblement supérieur du puits ne paraît guère postérieur au second tiers du IIIe siècle.

À première vue, l'éventuel rapport de ces structures, mises au jour en 1992, avec la fabrication des moules monétaires pourrait être soutenu, quand on sait que la cuisson des moules se pratiquait dans des fours assez sommaires ${ }^{11}$. Mais il faut bien admettre qu'un tel rapprochement ne s'impose pas d'emblée. En tout premier lieu parce que les moules n'ont pas été retrouvés «en situation». Difficile dans ces circonstances d'attacher les moules de Sées à un contexte d'utilisation déterminé. Pour autant, les observations archéologiques ne doivent pas faire écran. L'abondance des vestiges dans les remblais indique précisément que l'on se trouve au centre d'un quartier affecté à diverses spécialités artisanales. Dans le bruit de fond de ces activités, lier les moules découverts au sein du tissu urbain de la cité sagienne à l'existence d'une officine spécialisée dans le surmoulage de monnaies serait plausible. La seconde découverte effectuée en 1993 est plus probante, associant deux moules mais aussi des creusets à un édifice pourvu d'un four, son puits pouvant avoir été remblayé en fin d'activité. Pour des raisons de sécurité, il n'avait pas été possible de sonder plus avant ce puits et sans doute aurait-il pu nous apporter d'autres éléments démontrant une activité de surmoulage.

En la matière, les résultats d'autres fouilles ne manquent pas d'intérêt. Les vestiges d'installation destinée à la fabrication de monnaies par surmoulage sont notamment bien cernés par les fouilles récentes, à Lyon, d'une insula dévolue à

11. LaLlemand 1994, p. 143. Voir aussi les remarques de Andrieux et al. 2005, p. 225-252. 
l'habitat, au commerce et à l'artisanat ${ }^{12}$. Dans l'une des boutiques/ateliers ouverte sur la rue, les archéologues ont mis au jour onze moules monétaires en terre cuite et un faux denier de Trajan. Au moment de leur découverte, les moules et la monnaie étaient déposés dans une fosse interprétée comme un «cellier». Non moins important, la fosse-cellier, dont l'abandon se place vers le milieu du III siècle, était aménagée à quelques pas d'un foyer adossé à un mur. Quel sens donner à une telle configuration du lieu, qui paraît si proche de celui de la rue Amesland? Assurément que l'activité pratiquée dans la boutique/atelier accompagnait une chaîne opératoire complète, allant de la réalisation de moules (modelage et cuisson) à la fabrication de monnaies par surmoulage. Le même contexte caractérise des découvertes effectuées à Saint-Mard (Belgique). L'un des quartiers de l'agglomération antique a livré un ensemble particulièrement dense de moules monétaires du deuxième quart du IIIe siècle associés à des monnaies coulées ${ }^{13}$. Les divers déchets récoltés dans des dépotoirs et des fosses témoignent de l'installation, dans cette partie de la ville, d'un artisanat dédié à la métallurgie et au travail de l'os. De toute évidence, les trouvailles de moules monétaires prolongent les activités d'un quartier hautement spécialisé. Les découvertes de Châteaubleau (Seine-et-Marne) ne sont pas moins importantes. Elles nous renseignent sur la production sérielle vers 260 de monnaies dans de petites officines installées au cœur du vicus ${ }^{14}$. Principal constat : les objets recueillis in situ (flans, boudins, cylindres, moules à empreintes monétaires, etc.) ont permis de préciser les caractéristiques principales des officines et leurs productions, comme notamment l'association dans une même officine des techniques de coulage et de frappe. Ces installations témoignent manifestement d'une véritable organisation du monnayage d'imitations dans le vicus.

\section{Les moules du petit séminaire}

On ne serait pas exhaustif sans mentionner une autre découverte ; Sées est en effet réputé pour avoir livré peu après 1855 une vingtaine de moules lors du creusement de l'une des galeries du petit séminaire ${ }^{15}$ (actuelle basilique de l'Immaculée Conception). Une distance de $300 \mathrm{~m}$ sépare ce site de la rue Amesland (figure 1). À l'époque, la découverte attira l'attention de l'abbé Mallet, professeur au petit séminaire, qui prit soin d'en récupérer quelques exemplaires et en livra quelques informations consignées par le Bulletin de la Société historique et archéologique de l'Orne : «Ceux que possède le Petit-Séminaire sont de divers

12. CéCILlon et al. 2011, p. 57-59. Lyon est particulièrement réputé dans la littérature archéologique pour avoir livré, à plusieurs reprises depuis le XVIe siècle, des moules monétaires : cf. TurCan 1982, p. 9-29; Aubin, Monin 1996, p. 112-116.

13. Clausse 1994, p. 136-137.

14. Pilon 1998, p. 92 ; Pilon 2004, p. 235-261.

15. BERNOUIS 1999, p. 202. 
modules, grand, moyen et petit bronze. Il y en a vingt-deux aux effigies suivantes, les seules qu'on ait déchiffrées jusqu'ici : Nerva-Trajan (2), Hadrien (1), Antonin le Pieux (4), Commode (3), Septime-Sévère (1), Faustine (3) et Crispine (2) ${ }^{16} \gg$. Fait important : ces quelques lignes, directement transmises par l'abbé Mallet, comme l'indique une note de bas de page, décrivent des objets à face unique portant l'empreinte de monnaies de bronze du IIe siècle. En revanche, aucune information sur le contexte de découverte ne nous est parvenue. En d'autres termes, si de nombreuses zones d'ombre entourent l'origine archéologique des moules du petit séminaire, reste assurée l'émergence à Sées d'une pratique essentiellement tournée vers le surmoulage de bronzes du Haut-Empire.

Vers 1922, H. Sevray offrit quelques moules provenant de cette découverte à la Société historique et archéologique de l'Orne. Une enquête menée dans les réserves de la Société a permis de retrouver trois valves d'argile. L'examen montre qu'elles ont été obtenues à l'aide de revers de monnaies de bronze (tableau 1), dont les empreintes ont pu être comparées à celles de la rue Amesland. Aucune liaison n'a été mise en évidence et tout indique que ces moules ont été fabriqués à partir d'originaux différents. Seule la technique de production les rapproche. En effet, comme pour les moules de la rue Amesland, ils adoptent la forme d'une cupule empreinte d'un seul côté, pour une coulée qui s'effectuait vraisemblablement par paires de moules. Dans ces conditions, les découvertes de Sées ne seraient pas sans rappeler celles de l'agglomération antique de Châteaubleau (Seine-et-Marne) où plusieurs officines d'imitations ont fonctionné, peut-être au cours de périodes différentes, utilisant diverses techniques (frappe et coulage) et sans lien entre elles ${ }^{17}$.

\section{Le matériel et la fabrication des monnaies}

Les moules monétaires se présentent sous la forme d'un petit disque de $38 \mathrm{~mm}$ de diamètre en moyenne et de 0,8 à $10 \mathrm{~mm}$ d'épaisseur. Tous portent sur l'un des côtés d'une galette en matériau argilo-sableux l'empreinte en creux d'un droit ou d'un revers. L'autre côté de la galette est lisse et fortement bombé. La structure des pâtes, très fines, est caractéristique. On observe en effet une faible granulométrie, particulièrement adaptée à une plus grande finesse de réplique au moment de la prise d'empreinte. L'argile est beige et peut avoir viré au rouge/ orange, ce qui pourrait indiquer une atmosphère plus ou moins oxydante lors de la cuisson des moules. Au contact de la monnaie, de nombreux moules montrent également une zone grise, répartie de manière homogène sur l'ensemble de la surface. Cela s'explique probablement par la coulée d'un métal encore liquide et thermiquement homogène. 
Tout autour des empreintes, l'argile fait un ressaut de quelques millimètres, dont la surface supérieure marque une cassure. Le bord extérieur, quand il n'est pas endommagé, a été lissé et pourvu d'une excroissance perpendiculaire au disque, qui prend la forme d'un canal permettant la coulée du métal en fusion (figure 3).

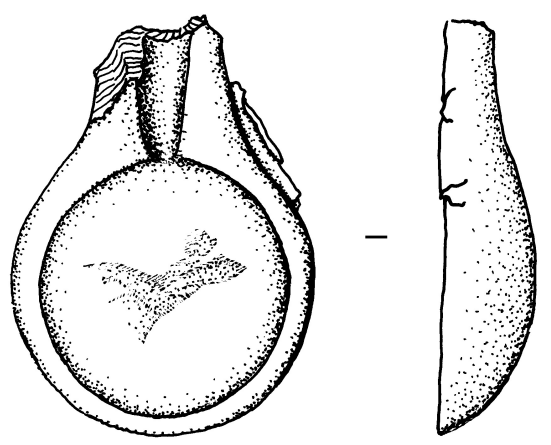

Figure 3 - Moule avec empreinte de revers d'un bronze du Haut-Empire découvert rue Amesland (dessin : Marie Pourrier ; échelle 1).

De nombreuses études ont été consacrées à la fabrication des moules monétaires. En ce sens, les travaux expérimentaux que mena H. Drescher, à partir des moules à couler des deniers et des bronzes des IIe-IIIe siècles découverts à Pachten (Allemagne), ont enrichi indéniablement notre connaissance du mode opératoire (figure 4$)^{18}$. La pratique la plus couramment rencontrée est celle qui consistait à faire alterner dans un tube métallique une galette d'argile, une monnaie, puis à nouveau une galette jusqu'à obtenir un rouleau où la plupart des galettes porteront une empreinte sur les deux faces (figure $4^{1}$ ). À ce stade de la préparation, la pile d'argile était extraite du cylindre et une encoche triangulaire était réalisée le long de la paroi, formant l'indispensable canal lors de la coulée du métal (figure $4^{2}$ ). Une par une, les galettes étaient alors démontées afin de récupérer les monnaies, puis la pile était remontée à l'identique (figure $4^{3}$ ). En renouvelant l'opération les piles étaient regroupées par deux ou trois ${ }^{19}$, l'encoche triangulaire $^{\prime}$ vers le centre, et enrobées dans une enveloppe d'argile (figure $4^{4}$ ). La coulée du métal en fusion pouvait commencer. Après refroidissement, il n'y avait pas

18. Drescher 1973, p. 95-99. Méthode reprise dans : Lallemand 1994, p. 143 ; Chameroy 2007, p. 533-536 ; CHAMEROY, GUIHARD 2014 (à paraître).

19. Les rouleaux se groupent par trois à Pachten et à Damery, tandis que l'association de deux rouleaux est attestée à Lingwell Gate ou encore à Whitchurch. Pour la bibliographie correspondante : R.-Alföldi 1971, pl. 5, no 12a-b ; Hiver de BeAuvoir 1837, p. 175 et pl. 6, no 15 ; AKerman 1838-1839, p. 156, n. 1 et fig. 5 ; Boon, Rath 1965, p. 38, fig. 7, no 1. 
d'autre solution que de briser l'ensemble pour récupérer les monnaies. Ainsi, dans un seul jet de coulée, une quantité plus ou moins importante de pièces pouvait être fabriquée, suivant le nombre de moules articulés entre eux. À Damery (Marne), par exemple, la découverte de rouleaux groupés par trois - de 13 moules chacun - indique une production allant jusqu'à une trentaine de monnaies ${ }^{20}$.

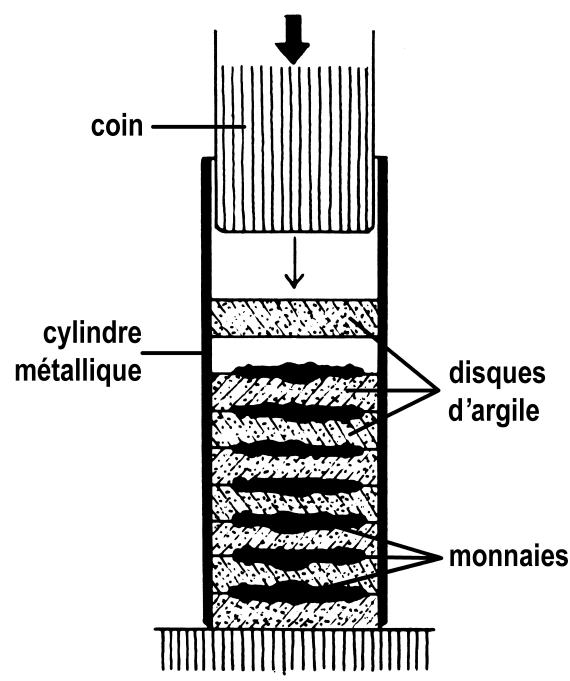

1

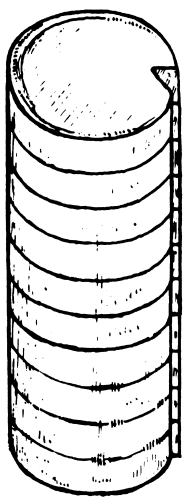

2

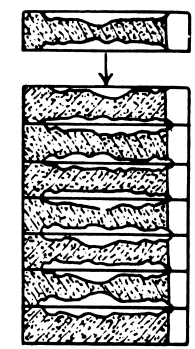

3

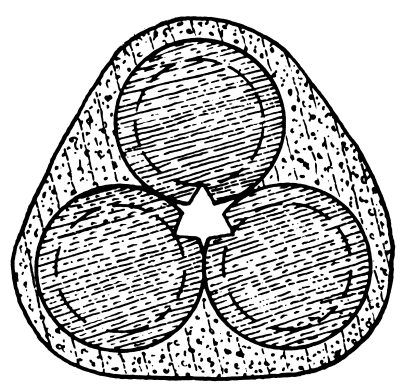

4

Figure 4 - Réalisation des moules monétaires d'argile (d'après DrEsCHER 1973).

20. Hiver (de Beauvoir) 1837, p. 175 et pl. 6, no 15. 
Les différences avec les moules de Sées paraissent sans appel. La forme de «poire» (figure 3), que leur confère le long canal de coulée prolongeant la rondelle d'argile, les distingue d'emblée ${ }^{21}$. En effet, nous avons souligné que les moules habituellement rencontrés en Gaule n'offrent en guise de canal de coulée qu'une petite entaille triangulaire. De plus, la technique mise en œuvre à Sées qui consistait à imprimer en creux, sur les deux disques d'argile présentant une face externe bombée, l'avers et le revers d'une monnaie officielle indique que ces disques ne s'empilaient pas pour être remplis en série, mais fonctionnaient à chaque fois par paire.

Partant de ces observations, le procédé suivi à Sées devait simplement consister à faire correspondre, par leurs faces imprimées, deux valves de droit et de revers, de sorte à former un moule individuel fermé. Toutefois, chaque paire pouvait composer les éléments d'une chaîne opératoire plus complexe. On peut notamment imaginer la réalisation d'un arbre de coulée pour garnir successivement les moules placés en chapelet ou côte à côte, permettant une production sérielle de monnaies. Quoi qu'il en soit, à la différence d'une articulation des moules en rouleau, qui, en une seule coulée, livraient plusieurs dizaines de monnaies, la technique utilisée à Sées était certainement bien loin d'apporter un haut rendement. Dès lors, on ne peut manquer de suggérer qu'il a pu s'agir d'une activité d'appoint, née d'un besoin très ponctuel et probablement limité dans le temps.

\section{Une officine de monnaies non officielles à Sées}

La technique du surmoulage et du coulage distingue d'emblée Sées des ateliers monétaires impériaux. En effet, ces derniers ont exclusivement recours à la frappe entre deux coins pour imprimer le droit et le revers sur les flans monétaires. En utilisant la coulée en lieu et place de la frappe, la pratique s'inscrit clairement à l'encontre des règles établies par le pouvoir officiel. Pourtant, la production de monnaies à partir de moules est bien attestée dans de petites officines locales, telles celles identifiées à Lyon, Saint-Mard ou encore Châteaubleau ${ }^{22}$. Étrangère au réseau des monetae publicae, l'officine sagienne a donc bel et bien produit des bronzes non officiels, mais s'agit-il, au regard de la loi, de fausses monnaies?

Rappelons qu'une monnaie est considérée comme officielle et authentique tant qu'elle n'a été manipulée ni dans sa substance métallique, ni lors de la frappe ; pour ce faire, elle doit avoir reçu la forma publica à partir de coins officiels, tandis que le poids de la pièce frappée ne définit pas en lui-même le caractère

21. La forme de poire est particulièrement originale pour ce genre d'objet et n'a été que très rarement rencontrée jusqu'ici : cf. notamment les deux moules monétaires signalés sur le sanctuaire de Népellier, dans Chameroy, Lambot 2011, p. 36-37.

22. Cf. supra 2.1. 
officiel d'une monnaie ${ }^{23}$. Le faux-monnayeur est, au départ, puni en vertu de la lex Cornelia de falsis (81 av. J.-C.) de diverses sanctions, allant de la croix à l'exil $^{24}$. Dans sa version actualisée sous Constantin (326), cette loi condamne expressément la technique du coulage de faux (si quis nummum falsa fusione formaverit), qui fait à l'évidence concurrence aux monetae publicae ${ }^{25}$. Ainsi, la réaction de Constantin, même tardive, à un phénomène probablement déjà ancien prouve que l'empereur ne pouvait accepter une telle activité dans l'Empire, mise clairement au rang de faux-monnayage.

Le surmoulage - exclusif - de monnaies de bronze à Sées pose toutefois un problème spécifique. Si la lex Cornelia de falsis condamne entre autres le coulage de fausses monnaies d'argent, elle ne se prononce pas sur le bronze ${ }^{26}$. Faut-il alors y reconnaître un vide juridique à l'égard des contrefaçons de bronze ? Pour C. King, la loi romaine ne les aurait pas considérées comme telles avant le Ive siècle ${ }^{27}$. Installés au sein du tissu urbain et au cœur d'un espace artisanal de la cité et dans un secteur ouvert, pour les découvertes de la rue Amesland, sur une voie de circulation, les monnayeurs de Sées n'ont, semble-t-il, guère été soucieux de cacher leur activité. Une telle situation topographique servirait dès lors d'argument à l'hypothèse suivie par plusieurs numismates d'une production tolérée au niveau local, mais non reconnue par le pouvoir officiel ${ }^{28}$. Nous y reviendrons en conclusion.

\section{La reconnaissance des matrices}

Comme nous l'avons remarqué, l'identification des moules commence, de façon naturelle, par une recherche des empreintes : quelles monnaies ont été utilisées pour la fabrication en série des moules ? Il nous faut maintenant aborder une autre question : combien de monnaies ont-elles été mobilisées au moment de la prise d'empreinte ? L'étude des matrices (ou «monnaies d'origine») est en cela déterminante, puisqu'elle éclaire, mieux qu'un simple décompte d'empreintes, sur le nombre de monnaies effectives dont disposait l'officine (figure 5). On peut raisonnablement estimer qu'une même pièce pouvait servir plusieurs fois, ne nécessitant pas de fait l'utilisation d'un nombre élevé de matrices. C'est ce

23. Wolters 1999, p. 370.

24. Pour un rappel des grandes lignes de la législation à ce sujet voir DeLmaIRE 1989, p. 502-503.

25. Grierson 1956, p. 252 et 255. Voir aussi Giardina 1973-1974, p. 184-190.

26. Wolters 1999, p. 364-365. Les dispositions prises au III' siècle concernant la fausse monnaie ne sont pas plus déterminantes. Dans les recueils de jurisconsultes, en particulier les «sentences» de Paul (Préfet du Prétoire en 222), la question de la fausse monnaie de bronze n'est pas envisagée. Voir également sur le sujet les commentaires de Besombes 2004, p. 35.

27. KING 1996, p. 246.

28. Schwartz 1975 ; Gricourt 1993, p. 564-566 ; Peter 2004, p. 26-29. Dans la région danubienne, les auteurs considèrent aussi les contrefaçons de bronze («Limesfalsa») comme une monnaie locale : voir KRÄNZL 1996, p. 268. 
que suggèrent d'ailleurs d'autres ensembles, comme ceux de Saint-Mard ${ }^{29}$ ou de La Coulonche ${ }^{30}$. Précisément, l'examen des moules de Sées nous montre que les monnayeurs disposaient d'un jeu d'au moins 19 bronzes (tableaux 2-3).

Parmi les droits, la monnaie la plus ancienne, identifiée avec certitude, est un dupondius de Domitien frappé à Rome entre 85 et 96 apr. J.-C. (tableau 2). On dénombre ensuite une majorité de monnaies des principats de Trajan et d'Hadrien, en tout 13 dupondii et as provenant de Rome et d'un atelier oriental. Enfin, deux dupondii romains, qui prennent place dans le quatrième consulat d'Antonin le Pieux (140-161), ont été utilisés.

Dans ce contexte, il est attendu que les matrices de revers portent en majorité l'empreinte de monnaies émises sous la dynastie des Antonins (tableau 3). Si naturelle que soit cette appréciation, elle se heurte pourtant à un problème. L'état fragmentaire du mobilier empêche en effet toute reconstruction des paires de moules qui aurait permis de relier les matrices de droit aux matrices de revers. Dès lors, il faudrait s'attendre à ce que plusieurs types de revers, communs à plusieurs empereurs ou à plusieurs émissions d'un même empereur, ne puissent être identifiés avec une grande précision. Toutefois, cette difficulté se révèle artificielle dans bien des cas. Le doute n'est guère permis pour plusieurs matrices de revers qui sont très caractéristiques par leur type - certains types n'étant connus que pour un règne en particulier. Ainsi, les matrices les plus précoces, au nombre de trois, peuvent être attribuées à une série orientale frappée sous Trajan en 116 et 117 . Il s'agit d'un type présentant les lettres SC dans une couronne de laurier. Au moins quatre dupondii présentant la Fortune assise et la légende FELICITATI AVG / / COS III P P au type du navire se rattachent, selon toute probabilité, au monnayage romain d'Hadrien des années 117-119 et 132-134. Les matrices les plus récentes renvoient, quant à elles, à des bronzes frappés à Rome au milieu du quatrième consulat d'Antonin le Pieux (145-161), dont quatre correspondent à la série TR POT XV COS IIII / / ANNONA AVG (151-152) au type de l'Annone. Les problèmes ont été plus compliqués pour cinq matrices (tableau 3, «E. ind. 1-5»). La mauvaise conservation des moules et le manque d'originalité des types identifiés - essentiellement des représentations symboliques de divinités - ont rendu délicate l'attribution à un monnayage en particulier. Pour illustrer la difficulté du classement, notons juste que les revers se retrouvent aussi bien sur des bronzes de Trajan et d'Hadrien que sur certains bronzes frappés au nom d'Antonin le Pieux.

$\mathrm{Au}$ final, les résultats auxquels nous parvenons sont très significatifs : en laissant de côté le bronze de Domitien, l'essentiel des matrices renvoie à des émissions des années 116-152. On ne saurait être plus précis. Et un tel resserrement ne pourrait s'expliquer que par le fait que les moules relèvent d'une unique phase de production. Un autre argument peut être avancé. Dans la majorité des cas,

29. LaLLEMAND 1994, p. 145.

30. Chameroy, Guihard 2014 (à paraître). 


\begin{tabular}{|c|l|c|}
\hline \multicolumn{2}{|l|}{} & Dénomination \\
\hline \multirow{2}{*}{ Domitien } & $\begin{array}{l}\text { IMP CAES DOMIT AVG GERM COS... } \\
\text { Buste radié à dr. avec aegis. }\end{array}$ & Dupondius \\
\hline
\end{tabular}

\begin{tabular}{|c|c|c|}
\hline \multirow{2}{*}{ Trajan } & $\begin{array}{l}\text { IMP CAES NERVAE TRAIANO AVG GER DAC P M TR P COS V (ou VI) P P } \\
\text { Tête radiée à dr. } \\
\text { IMP CAES NER TRAIANO OPTIMO AVG GER DAC P M TR P COS VI P P } \\
\text { Buste radié avec égide sur l'épaule g., vu de trois quarts en avant. }\end{array}$ & Dupondius \\
\hline & $\begin{array}{l}\text { IMP CAES NER TRAIANO OPTIMO AVG GERM } \\
\text { Buste radié avec égide sur l'épaule g., vu de trois quarts en avant. }\end{array}$ & As \\
\hline
\end{tabular}

\begin{tabular}{|l|l|c|}
\hline \multirow{4}{*}{ Hadrien } & $\begin{array}{l}\text { IMP CAESAR TRAIAN(VS) HADRIANVS AVG P M TR P COS III } \\
\text { Buste radié à dr. et drapé sur l'épaule g. }\end{array}$ & Dupondius \\
\cline { 2 - 4 } & $\begin{array}{l}\text { IMP CAESAR TRAIAN HADRIANVS AVG } \\
\text { Tête laurée à dr. }\end{array}$ & As \\
\cline { 2 - 4 } & $\begin{array}{l}\text { IMP CAESAR TRAIANVS HADRIÁ à dr. et drapé sur l'épaule g. } \\
\text { HADRIANVS AVGVSTVS } \\
\text { Tête radiée à dr. }\end{array}$ & Dupondius \\
\cline { 2 - 4 } & $\begin{array}{l}\text { HADRIANVS AVG[...] } \\
\text { Buste lauré à dr. }\end{array}$ & As \\
\cline { 2 - 4 } & Indéterminé & Matrice ind. \\
\hline
\end{tabular}

\begin{tabular}{|l|l|c|}
\hline \multirow{2}{*}{ Antonin le Pieux } & $\begin{array}{l}\text { ANTONINVS AVG PI-VS P P TR P }[\ldots] \\
\text { Tête radiée à dr. }\end{array}$ & $\begin{array}{l}\text { Dupondius } \\
\text { IMP CAES T AEL HADR ANTONINVS AVG PIVS P P } \\
\text { Tête radiée à dr. }\end{array}$ \\
\cline { 2 - 4 } & Indéterminé & Matrice ind. \\
\hline
\end{tabular}

\begin{tabular}{|l|r|l|}
\hline $\begin{array}{l}\text { Dynastie } \\
\text { antonine }\end{array}$ & As ou Dupondius \\
\hline & Nombre total minimal des matrices de $\mathbf{D} /$ & \\
\hline
\end{tabular}




\begin{tabular}{|c|c|c|c|l|}
\hline Matrice & $\begin{array}{c}\text { Rue Amesland } \\
\text { [Musée Normandie (D.2005.13)] }\end{array}$ & $\begin{array}{c}\text { Datation } \\
\text { empreinte }\end{array}$ & Atelier & Référence \\
\hline D 1 & 1 & $85-96$ & Rome & RIC II, p. 191-206. \\
\hline
\end{tabular}

\begin{tabular}{|c|c|c|c|c|}
\hline $\begin{array}{l}\text { T } 1 \\
\text { T } 2 \\
\text { T } 3\end{array}$ & $\begin{array}{c}10 \\
15 ; 34 \\
56\end{array}$ & $\begin{array}{l}103-117 \\
114-116\end{array}$ & Rome & $\begin{array}{l}\text { Besombes } 225-233 \text { et } 716-719 \text {; } B M C \text { III, p. } 186- \\
225 \text {; RIC II, p. } 277-286 \text {; Strack 369, 378, } 382 . \\
\text { Besombes } 851 \text {; BMC III, - ; RIC - ; Strack } 455 .\end{array}$ \\
\hline $\mathrm{T} 4$ & $3 ; 24$ & $116-117$ & Asie & $\begin{array}{l}\text { Besombes 956-957 ; BMC III, p. } 232 \text {; } \\
\text { RIC II, } 644 \text {; Strack } 479 .\end{array}$ \\
\hline T 5 & 9 & & & \\
\hline T 6 & 11 & & & \\
\hline
\end{tabular}

\begin{tabular}{|c|c|c|c|c|}
\hline $\begin{array}{l}\mathrm{H} 1 \\
\mathrm{H} 2\end{array}$ & $\begin{array}{l}5 \\
7\end{array}$ & $119-121$ & & BMC III, p. 419-422 ; RIC II, p. 413-418. \\
\hline H 3 & 42 & $119-121$ & & BMC III, p. 423-425; RIC II, p. 420. \\
\hline H 4 & $45 ; 54$ & 119 & Rome & BMC III, 1167-1168 ; RIC II, 570-571. \\
\hline H 5 & 22 & $125-128$ & & BMC III, p. 435 et suiv. ; RIC II, p. 423 et suiv. \\
\hline H 6 & 52 & & & \\
\hline H 7 & 8 & $125-138$ & & \\
\hline Matrice ind. & 20 & & & \\
\hline
\end{tabular}

\begin{tabular}{|c|c|c|c|c|}
\hline $\begin{array}{l}\text { A } 1 \\
\text { A } 2\end{array}$ & $4 ; 25 ; 28 ; 19 ; 16$ & $\begin{array}{c}140 / 145-161 \\
151-152\end{array}$ & Rome & $\begin{array}{l}\text { BMC IV, p. } 280-281 \text {; RIC III, p. } 113 \text { et suiv. } \\
\text { BMC IV, } 1897 \text {; RIC III, } 895-898 .\end{array}$ \\
\hline Matrice ind. & 2 & & & \\
\hline
\end{tabular}

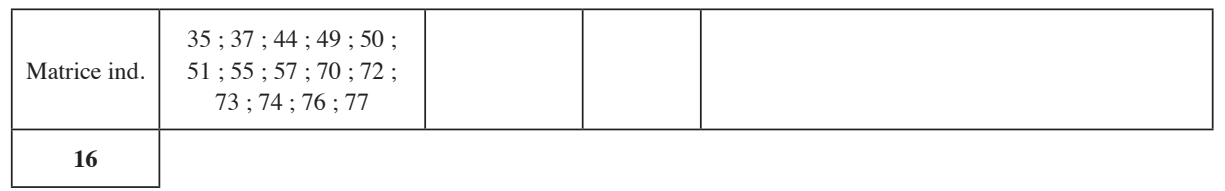

Tableau 2 - Répartition des moules et fragments d'après les matrices de droit. 


\begin{tabular}{|c|l|c|c|}
\hline \multicolumn{2}{|l|}{} & Dénomination & Matrice \\
\hline \multirow{2}{*}{ Trajan } & $\begin{array}{l}\text { DAC PARTHICO P M TR POT XX COS VI P P } \\
\text { Type SC dans une couronne de laurier }\end{array}$ & As & T 1 \\
& & & T 2 \\
\hline
\end{tabular}

\begin{tabular}{|l|l|r|r|}
\hline \multirow{3}{*}{ Hadrien } & $\begin{array}{l}\text { Type Fortune assise à g., tenant un gouvernail de la main dr. } \\
\text { et une corne d'abondance de la main g. }\end{array}$ & Dupondius & H 2 \\
& FELICITATI AVG // COS III P P & H 3 \\
\hline
\end{tabular}

\begin{tabular}{|l|l|c|c|}
\hline & $\begin{array}{l}\text { TR POT XV COS IIII / / ANNONA AVG } \\
\text { Type Annone assise à g., tenant deux épis de blé de la main dr. } \\
\text { au-dessus d'un modius et une corne d'abondance de la main g. }\end{array}$ & A 1 \\
Antonin le Pieux & $\begin{array}{l}\text { A } 2 \\
\text { Bype } \text { Britannia assise à g. sur un rocher, soutenant sa tête de la main dr. } \\
\text { et tenant un vexillum transversal de la main g. ; devant bouclier rond } \\
\text { AETER-NITAS (pour Faustine) } \\
\text { Type Providence debout à g., tenant un globe de la main dr. et un } \\
\text { sceptre de la main g. } \\
\text { AETER-NITAS (pour Faustine) } \\
\begin{array}{l}\text { Type Juno (?), debout à dr., levant la main g. } \\
\text { et laissant près du corps la main dr. }\end{array}\end{array}$ & A 5 \\
\hline
\end{tabular}

\begin{tabular}{|c|l|c|c|}
\hline & $\begin{array}{l}\text { Type Espérance avançant vers la g., tenant une fleur de la main dr. } \\
\text { et relevant le bas de sa robe de la main g. } \\
\text { Type Fortune debout à g., tenant un gouvernail de la main dr. } \\
\text { et une corne d'abondance de la main g. } \\
\begin{array}{l}\text { Dynastie } \\
\text { antonine }\end{array}\end{array}$ & $\begin{array}{l}\text { Type Fidélité debout à dr., tenant de la main dr. des épis de blé } \\
\text { orientés vers le bas et un plateau de fruits de la main g. } \\
\text { Type Junon assise à g., tenant une patère de la main dr. }\end{array}$ & E.ind 1 \\
\cline { 2 - 5 } & $\begin{array}{l}\text { Type Personnage debout à g., tenant une corne d'abondance } \\
\text { de la main dr. } \\
\text { [...] III }\end{array}$ & $\begin{array}{c}\text { As ou } \\
\text { Dupondius }\end{array}$ & E.ind 3 \\
\hline
\end{tabular}

\begin{tabular}{|l|c|c|c|}
\hline Type ind. & & $\begin{array}{c}\text { As ou } \\
\text { Dupondius }\end{array}$ & Matrice ind. \\
\hline \multicolumn{3}{|c|}{ Nombre total minimal des matrices de R/ } & $\mathbf{1 9}$ \\
\hline
\end{tabular}




\begin{tabular}{|c|c|c|c|l|}
\hline $\begin{array}{c}\text { Rue Amesland } \\
\text { [Musée Normandie (D.2005.13)] }\end{array}$ & $\begin{array}{c}\text { Petit séminaire } \\
\text { [Société historique et } \\
\text { archéologique de l'Orne] }\end{array}$ & $\begin{array}{c}\text { Datation } \\
\text { empreinte }\end{array}$ & Atelier & Référence \\
\hline $36 ; 60 ; 71$ & & $116-117$ & Asie & $\begin{array}{l}\text { Besombes 953-961; BMC III, p. 232-233; 646-648; Strack 479. } \\
38\end{array}$ \\
$59 ; 53 ; 6$ & & & \\
\hline
\end{tabular}

\begin{tabular}{|c|c|c|c|c|}
\hline 58 & & $117-119$ & & \\
$61 ; 65$ & & Rome & \\
$18 ; 63 ; 26 ; 78$ & & $132-134$ & & BMC III, p. 460-461; RIC II, p. 433 \\
48 & & & \\
\hline
\end{tabular}

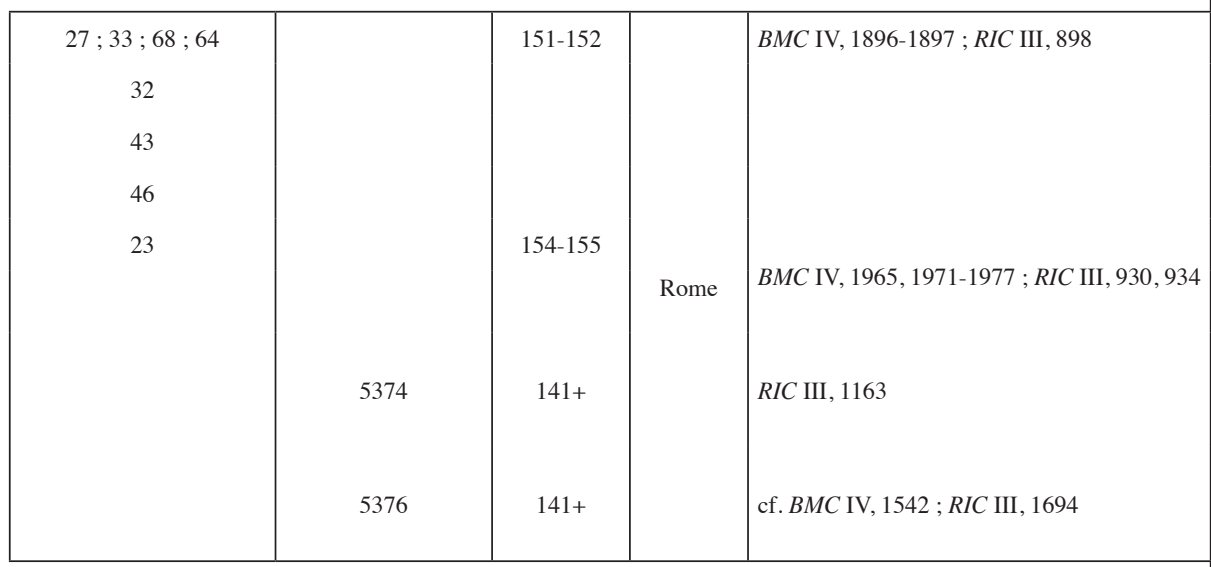

\begin{tabular}{|c|c|c|c|c|}
\hline 39 & & & & \\
47 & & & & \\
$12 ; 13 ; 41$ & & & & \\
31 & 5378 & & & \\
& & & & \\
\hline $\begin{array}{c}14 ; 21 ; 29 ; 30 ; 62 ; \\
66 ; 67 ; 69 ; 75\end{array}$ & & & & \\
\hline
\end{tabular}

Tableau 3 - Répartition des moules et fragments d'après les matrices de revers. 
le type de droit et le module des monnaies utilisées pour la confection des moules sont toujours les mêmes : une tête radiée et un diamètre large. Tout indique semble-t-il que ces monnaies (ou matrices) ont été prélevées au même moment et peut-être dans la circulation courante du site. S'il y eut donc une officine à Sées, son activité dut probablement être très limitée dans le temps afin de répondre à un besoin spécifique et en faisant avec les monnaies alors disponibles.

Faut-il pour autant ancrer strictement l'activité de celle-ci dans le IIe siècle ? Une réponse par l'affirmative serait un peu courte ${ }^{31}$. Elle contribuerait à donner trop d'importance à une lecture étroite et au pied de la lettre des dates d'émission des matrices. Depuis les travaux de Th.V. Buttrey ${ }^{32}$, nous savons que les sesterces, dupondii et as du Haut-Empire ne doivent être utilisés comme éléments de datation qu'avec la plus grande prudence. Ces questions chronologiques ne sont pas secondaires et doivent être examinées dans le détail. Elles permettront de mieux comprendre le développement d'une activité de surmoulage à Sées.

\section{Chronologie et sens de la production}

Précisément, l'état de conservation des monnaies qui ont servi à prendre les empreintes s'inscrit en faux contre une application mécaniste des dates d'émission pour situer la phase d'activité de l'officine. Toutes les matrices qui ont été utilisées par les faussaires présentent un degré d'usure important, correspondant plus ou moins à l'ancienneté de leur frappe. Celles de Domitien, Trajan et Hadrien sont fort usées, à l'exception peut-être de certaines matrices relevant du règne d'Hadrien. À partir d'Antonin le Pieux, les monnaies sont en bien meilleur état. Seule l'empreinte de droit A1 semble faire exception, ainsi que les revers A3-5 (figure 5).

On lira sans peine dans ce fait l'utilisation de monnaies qui n'étaient pas d'une grande fraîcheur de frappe et qui ont été vraisemblablement prélevées dans la circulation courante du site. Comparée à d'autres découvertes régionales, cette précision constitue un indice chronologique d'importance. En effet, nous savons que sur nos sites circulaient pendant le III siècle des bronzes du HautEmpire à l'usure très prononcée ${ }^{33}$. Loin d'être de simples curiosités numismatiques, ces trouvailles ajoutent beaucoup aux observations précédentes et, d'une certaine façon, éclairent la période pendant laquelle l'officine sagienne a fonctionné. De fait, on notera que l'édifice pourvu d'un four et d'un puits et fouillé en 1993 semble principalement datable des deux premiers tiers du IIIe siècle, à en juger par les mobiliers céramiques qu'il a livrés.

31. Contra Bernouis 1999, p. 199.

32. ButTREy 1972, p. 33-58.

33. Les découvertes monétaires de l'Espace du Palais et de la place Foch à Rouen démontrent l'utilisation prolongée dans la circulation régionale de grands et moyens bronzes des Ier et II siècles : cf. Hollard, Pilon 2005-2006, p. 61. 
Domitien, matrice de droit

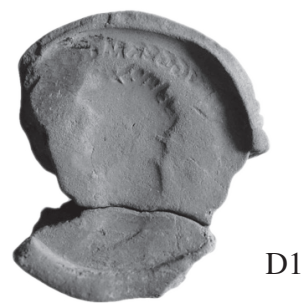

Figure 5a - Les moules de la rue Amesland (musée de Normandie D.2005.13).

Trajan, matrices de droit
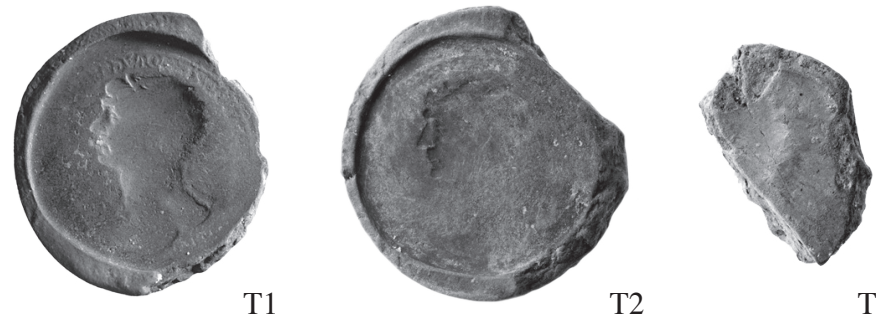

$\mathrm{T} 1$

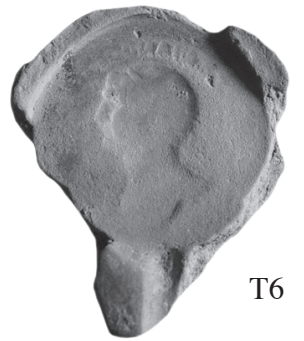

$\mathrm{T} 3$

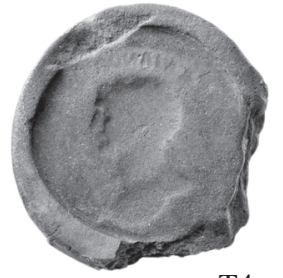

$\mathrm{T} 4$
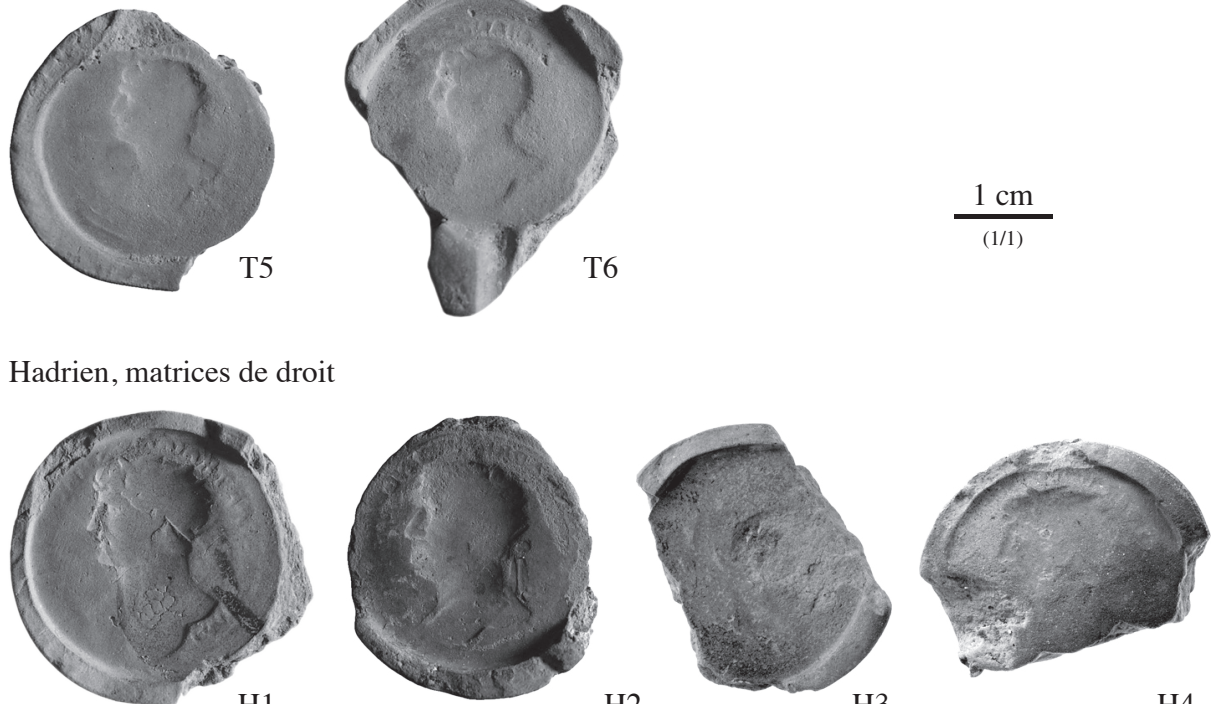

H1

$\mathrm{H} 2$

H3

H4
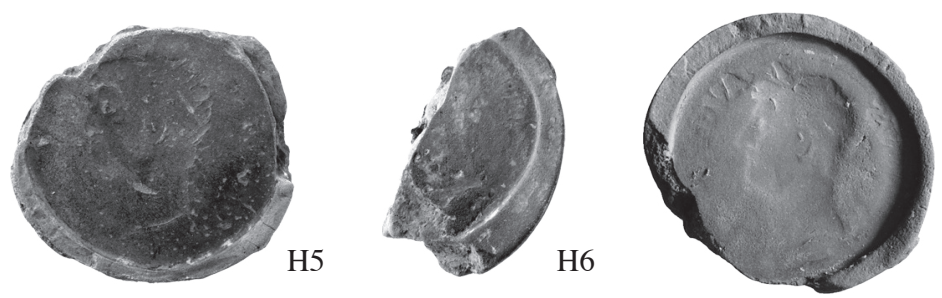

$\mathrm{H} 7$ 
Antonin le Pieux, matrices de droit
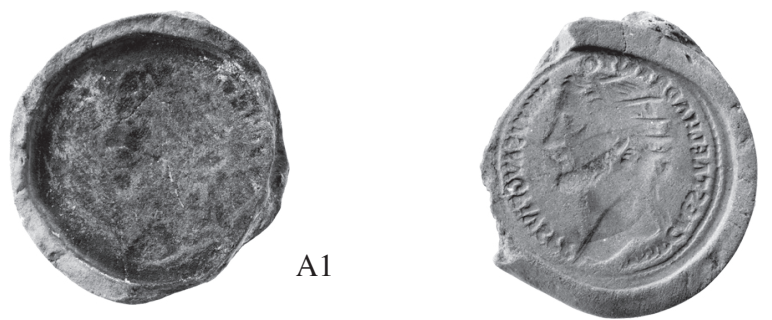

A2

Trajan, DAC PARTHICO P M TR POT XX COS VI P P, matrices de revers
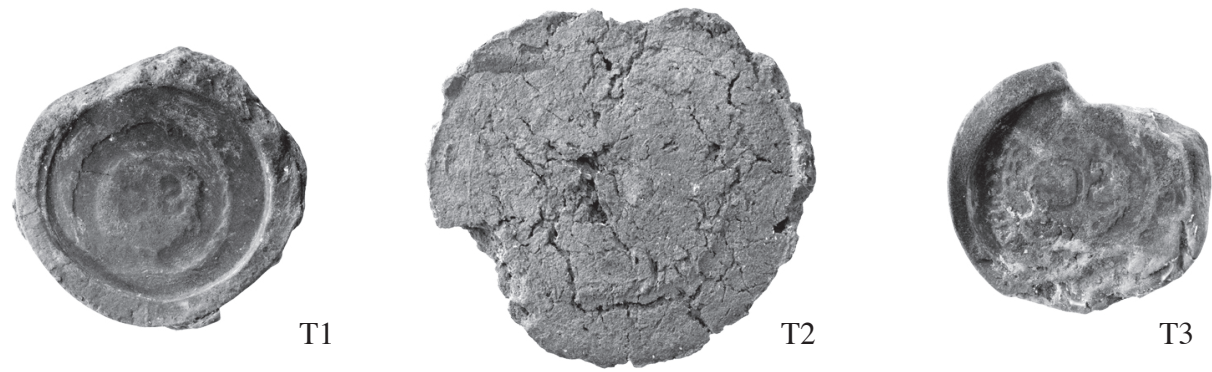

$\mathrm{T} 1$

$\mathrm{T} 2$

T3

Hadrien, Fortune assise à g., tenant un gouvernail et une corne d'abondance, matrices de revers

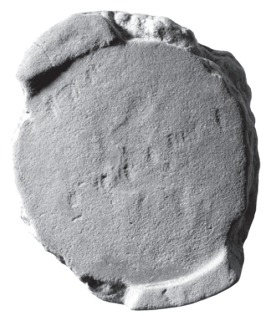

H1

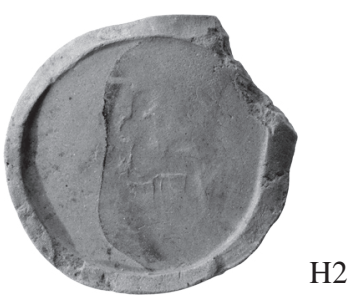

Hadrien, FELICITATI AVG // COS III P P, matrices de revers
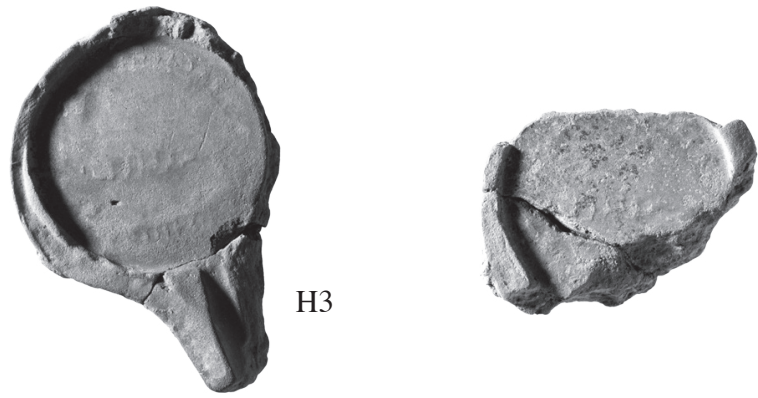

$\frac{1 \mathrm{~cm}}{(1 / 1)}$

Figure 5b - Les moules de la rue Amesland (musée de Normandie D.2005.13). 
Antonin le Pieux, TR POT XV COS IIII // ANNONA AVG, matrices de revers

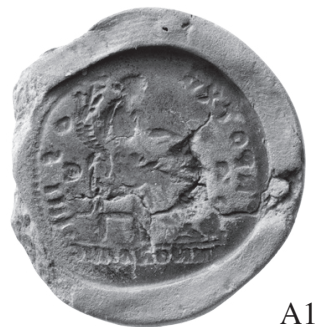

A1

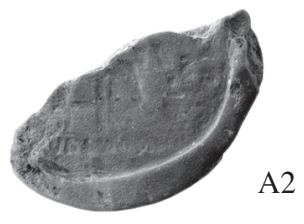

A2

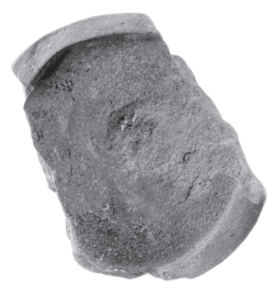

A3

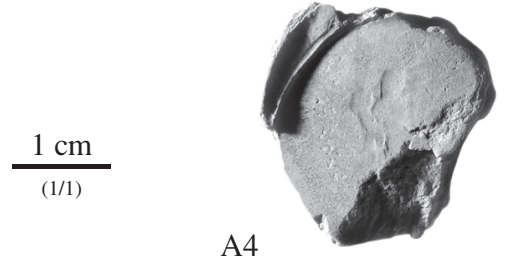

A4

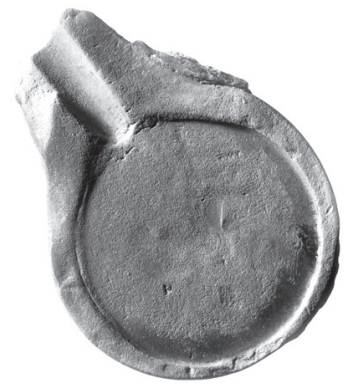

A5

Dynastie antonine, Espérance avançant vers la g., tenant une fleur et relevant le bas de sa robe, matrice de revers

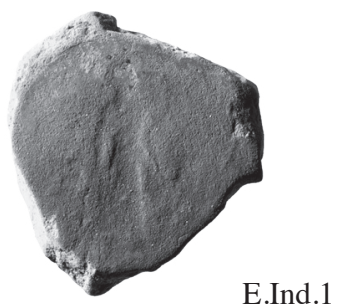

Dynastie antonine, Fidélité debout à dr., tenant des épis de blés orientés vers le bas et un plateau de fruits, matrice de revers
Dynastie antonine, Fortune debout à g., tenant un gouvernail et une corne d'abondance, matrice de revers

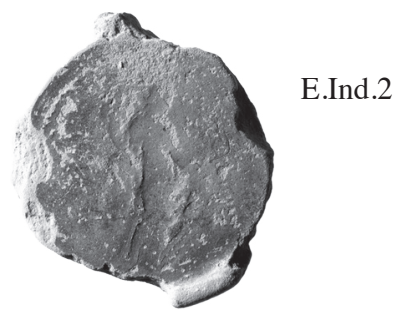

Dynastie antonine, Junon assise à g., tenant une patère, matrice de revers
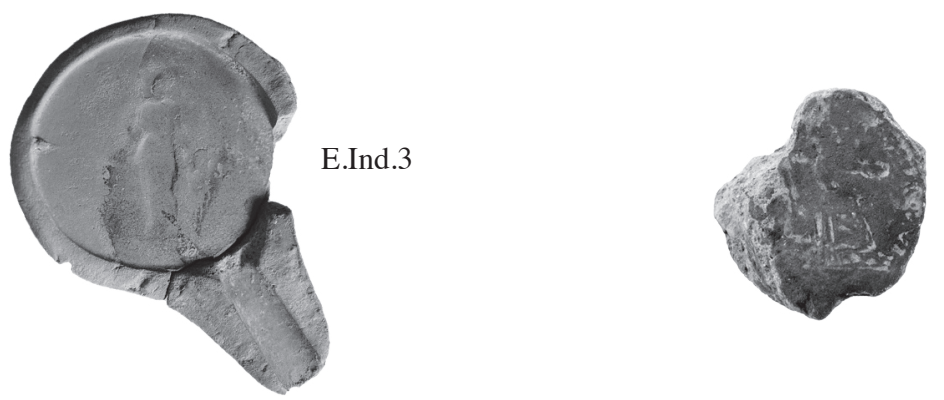

E.Ind.4

Figure 5c - Les moules de la rue Amesland (musée de Normandie D.2005.13). 
Antonin le Pieux pour Faustine, AETER-NITAS,

Providence debout à g., tenant un globe de la main dr., et un sceptre de la main g., matrice de revers

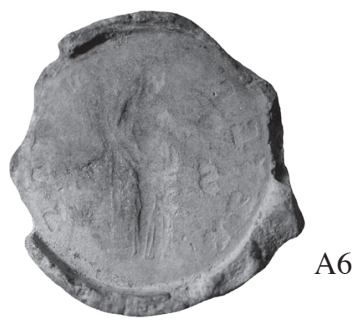

$\frac{1 \mathrm{~cm}}{(1 / 1)}$
Antonin le Pieux pour Faustine, AETER-NITAS, Juno (?), debout à dr., levant la main g. et laissant près du corps la main dr., matrice de revers
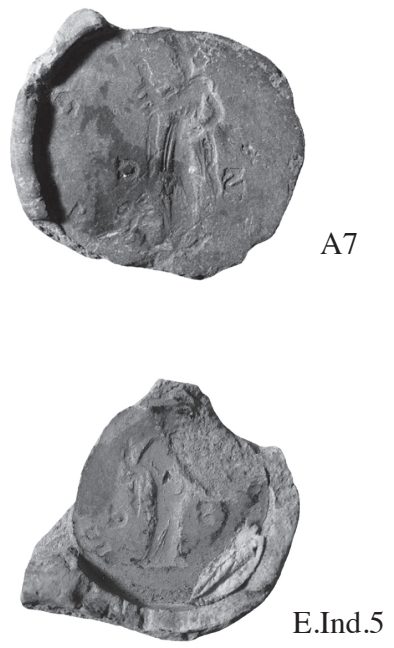

Figure 5d - Les moules du Petit séminaire, église de l'Immaculée Conception (Arch. dép. de l’Orne)

\section{La circulation du bronze en Gaule septentrionale au milieu du IIIe siècle}

À vrai dire, poser comme hypothèse de travail qu'au moment de la confection des moules les matrices avaient déjà connu une longue circulation n'a rien de particulièrement original.

Grâce aux larges enquêtes de J.-P. Callu, on connaît bien la circulation du bronze en Occident. En 1969 il en a exposé les grandes lignes ${ }^{34}$, en soulignant que la monnaie de bronze ne jouait plus au III siècle le rôle impérial qui avait été le sien au cours du siècle précédent. Elle était devenue une monnaie locale, limitée à l'Italie, à l'Espagne, et à l'Afrique du Nord ${ }^{35}$. Plus on s'éloignait de ces régions et plus les provinces eurent de grandes difficultés à s'approvisionner en bronzes frappés à Rome. En Gaule, tout comme en Britannia ${ }^{36}$ ou encore dans les Germanies, les monnaies de bronze émises après le règne de Commode

34. Callu 1969, p. 111-146. Sur la circulation du bronze voir aussi Thirion 1960 ; Buttrey 1972, p. 45-48.

35. Bost et al. 1992, p. 103-116 ; BernardelLi 2006, p. 69-101.

36. Voir notamment ABDy 2006, p. 45-55. 
(180-192) y circulaient peu et surtout le stock en ce métal a vu survivre en abondance les émissions des générations passées ${ }^{37}$. Ainsi, il apparaît que la plupart des trésors des deux premiers tiers du III siècle montre une composition analogue ${ }^{38}$. On constate non seulement que l'essentiel des émissions se rattachent au IIe siècle, mais aussi que la répartition chronologie est régulière. Les espèces de Trajan représentent en général $c a .10 \%$ des ensembles, celles d'Hadrien, d'Antonin le Pieux et de Marc Aurèle de 20 à $30 \%$ pour chacun des empereurs. L'abondance du II siècle (en moyenne $90 \%$ ) se clôt par les émissions de Commode. Fait tout aussi significatif, les espèces du Ier siècle demeurent toujours très discrètes ${ }^{39}$. P. Bastien a également étudié les développements de ce phénomène ${ }^{40}$. Les renseignements qu'il nous apporte, à travers l'étude minutieuse de nombreux dépôts monétaires, ne sont pas négligeables. Si la thésaurisation du bronze commence dès le Ier siècle, elle s'accentue fortement au début de la seconde moitié du ine siècle. Les trésors se composent alors de sesterces, dupondii et as émis de Trajan à Commode. Aux monnaies du Haut-empire se mêlent également des espèces frappées par Postume et sous le règne conjoint de Gallien et Valérien. Avec l'avènement d'Aurélien, les bronzes disparaissent significativement des dépôts monétaires.

Par conséquent, tout se passerait comme si la circulation du bronze en Gaule au IIIe siècle n'avait principalement joué que sur les espèces du Haut-Empire, traduisant une certaine sclérose des circuits monétaires. L'explication la plus habituelle, et qui paraitt la plus déterminante, serait d'ordre économique. À partir des années 240, l'antoninien, devenu l'espèce dominante du numéraire impérial, subit une dégradation rapide, à la fois en termes de poids et d'aloi ${ }^{41}$. Certains auteurs ont parlé à son sujet d'une "érosion de l'unité de compte monétaire ${ }^{42}$ ». Parallèlement, comme le laisse entrevoir la composition des trésors du tableau 4, la Gaule subsistait sur un capital monétaire non renouvelé. Depuis le début du III' siècle, l'atelier de Rome, où les émissions de sesterces, as et dupondii s'étaient considérablement réduites, n'exportait que peu de bronzes au-delà des Alpes ${ }^{43}$. Comme l'a bien souligné R. Turcan ${ }^{44}$, dans son étude du trésor de Guelma, Septime Sévère et ses successeurs avaient fait le choix d'augmenter la circulation de la monnaie d'argent et de restreindre celle du bronze. La situation avait donc toutes les chances d'être préoccupante. Elle constituait une

37. Hollard 1996, p. 211 ; Hollard, Foucray 1995, p. 74.

38. Voir l'analyse des trésors de France et de Belgique réalisée par DesNier 1985, p. 35-39.

39. Certains auteurs y ont vu les effets d'une refonte presque généralisée : cf. ETIENNE, RACHET 1984.

40. B ASTIEN 1967, p. 95-108, en part. 106-108.

41. Callu 1969, p. 237-249. Voir aussi Depeyrot, Hollard 1987.

42. Hollard 1992, p. 74.

43. CALlu 1969, p. 115-119.

44. TURCAN 1963, p. 50-53. 
menace sérieuse de pénurie en petit numéraire, puisque, pour l'appoint dans les transactions privées et les besoins locaux, le bronze restait très utile ${ }^{45}$. À l'évidence, les mutations qui affectaient les monnaies d'argent et l'arrêt de l'approvisionnement de la Gaule en bronzes frais sont autant d'éléments déterminants pour comprendre les raisons qui ont entraîné une thésaurisation et une utilisation prolongée des bronzes du Haut-Empire.

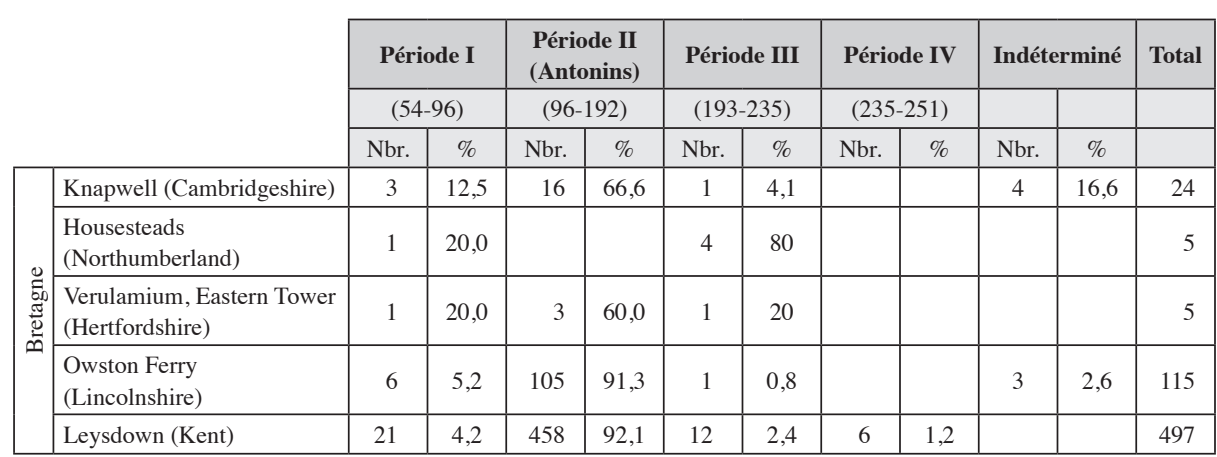

\begin{tabular}{|l|l|r|r|r|r|r|r|r|r|r|r|}
\hline Solre-Saint-Géry (Belgique) & & & 24 & 92,3 & 2 & 7,6 & & & & & 26 \\
\hline Morialmé (Belgique) & 4 & 2,9 & 124 & 90,5 & 7 & 5,1 & 2 & 1,4 & & & 137 \\
\hline Manage (Belgique) & 9 & 3,5 & 237 & 93,6 & 6 & 2,3 & & & 1 & 0,3 & 253 \\
\hline Froidmont (Belgique) & 8 & 5,6 & 127 & 90,0 & 1 & 0,7 & & & 5 & 3,5 & 141 \\
\hline Flobecq (Belgique) & 2 & 2,1 & 91 & 95,7 & 2 & 2,1 & & & & & 95 \\
\hline Mélin (Belgique) & 13 & 5,2 & 230 & 92,0 & 5 & 2 & & & 2 & 0,8 & 250 \\
\hline Beveren-Leie (Belgique) & 19 & 4,1 & 435 & 94,9 & 3 & 0,6 & 1 & 0,2 & & & 458 \\
\hline Bourg-Blanc (Finistère) & 27 & 2,2 & 1125 & 93,7 & 36 & 3 & 12 & 1 & & & 1200 \\
\hline Vilaines (Sarthe) & 9 & 3,4 & 225 & 86,5 & 6 & 2,3 & 20 & 7,6 & & & 260 \\
\hline $\begin{array}{l}\text { Marboué «Annouillet» } \\
\text { (Eure-et-Loir) }\end{array}$ & 3 & 0,5 & 507 & 93,0 & 29 & 5,3 & 6 & 1,1 & & & 545 \\
\hline Chilleurs-aux-Bois (Loiret) & 27 & 4,1 & 599 & 91,7 & 19 & 2,9 & 8 & 1,2 & & & 653 \\
\hline $\begin{array}{l}\text { Arnouville-Lès-Gonesse } \\
\text { (Val-d'Oise) }\end{array}$ & 98 & 4,09 & 2218 & 92,6 & 69 & & 1 & & 7 & & 2393 \\
\hline Epiais-Rhus (Val-d'Oise) & 17 & 3,3 & 475 & 93,6 & 11 & 2,1 & 4 & 0,7 & & & 507 \\
\hline La Groutte (Cher) & 13 & 7,2 & 160 & 89,3 & 6 & 3,3 & & & & & 179 \\
\hline Primelles (Cher) & 1 & 0,5 & 179 & 95,7 & 6 & 3,2 & & & 1 & & 187 \\
\hline Bourges 7 (Cher) & 4 & 1,1 & 336 & 92,8 & 13 & 3,5 & 6 & 1,6 & 3 & 0,8 & 362 \\
\hline Sainte-Montaine (Cher) & & & 81 & 92,0 & & & 2 & 2,2 & 5 & 5,6 & 88 \\
\hline Clémont-sur-Sauldre (Cher) & & & 84 & 97,6 & & & 2 & 2,3 & & & 86 \\
\hline Puy-Dieu (Haute-Vienne) & 30 & 3,86 & 702 & 90,2 & 35 & 4,4 & 11 & 1,4 & & & 778 \\
\hline Bordeaux (Gironde) & 3 & 3,4 & 82 & 95,3 & 1 & 1,1 & & & & & 86 \\
\hline
\end{tabular}

Tableau 4 - Trésors de bronzes se terminant par des émissions postérieures à la Dynastie des Antonins.

45. Callu 1969, p. 125. 
C'est vraisemblablement pour répondre à ces fortes tensions sur le numéraire de faible valeur qu'il faut dès lors situer l'activité de l'officine sagienne. Nous écartons ici une comparaison avec les Limesfalsa. La circulation monétaire est en effet tout autre sur le Danube. Comme l'a montré l'étude de M. Pfisterer, l'approvisionnement en bronzes post Antonins est plus régulier et ne peut à ce titre servir de jalon chronologique pour dater la production de bronzes coulés à Sées $^{46}$. Quand on reconnaît que la majorité des monnaies utilisées pour le surmoulage sont des bronzes du IIe siècle (as et dupondii de Trajan à Antonin le Pieux), quand, de surcroît, on se rappelle l'importance de leur usure, comment ne pas en déduire que les moules de Sées s'inscrivent tout à fait dans le contexte monétaire gaulois du second tiers du IIIe siècle ? Leur contexte de découverte renforce cette hypothèse chronologique. Mais plus encore : que cette appréciation soit plausible, nous croyons pouvoir le démontrer en portant, dès à présent, notre attention sur la structure des trésors de bronze en Gaule septentrionale.

\section{La structure des dépôts}

Dans le vaste ensemble géographique défini, la relative abondance au IIIe siècle des bronzes du Haut-Empire montre qu'ils étaient destinés à circuler largement et, de fait, du Centre au Nord de la Gaule, ils sont présents. L'actuelle Normandie ne fait pas exception. Treize trésors se terminant par des émissions postérieures aux Antonins ont pu être identifiés (figure 6) ${ }^{47}$ :

- Anvéville (Seine-Maritime) - Découverte en 1844 dans une amphore en bronze de 200 monnaies de Nerva, Trajan, Hadrien, Antonin le Pieux, Faustine mère et fille, Marc Aurèle, Commode et Sévère (Septime Sévère ou Alexandre Sévère ?).

- Esclavelles (Seine-Maritime) - Découverte en 1835 dans un vase en terre noire de 380 monnaies de Trajan, Hadrien, Antonin le Pieux, Faustine, Marc Aurèle, Commode et Septime Sévère.

- Sainte-Beuve-en-Rivière (Seine-Maritime) - Découverte en 1809 dans plusieurs vases de 4000 monnaies d'Auguste, Tibère, Néron, Vespasien, Domitien, Trajan, Hadrien, Sabine, Aelius, Antonin le Pieux, Marc Aurèle, Lucius Verus, Faustine mère et fille, Commode, Lucille, Septime Sévère, Julia Domna, Caracalla, Geta, Julia Aquilia Severa, Julia Mamaea, Maximin, Philippe père et fils.

- Conches-en-Ouche (Eure) - Découverte vers 1867 de 134 grands bronzes de Jules César (?), Néron, Trajan et Hadrien jusqu'à Gordien III.

46. Pfisterer 2007, p. 672 et suiv.

47. Trouvailles inventoriées dans Loriot, ScheERs 1985, p. 20 (Anvéville), 29 (Esclavelles), 77 (Conches-en-Ouche et Les Damps), 78 (Dardez), 80 (Évreux I), 91 (Rouge-Perriers); Blanchet 1900, p. 187-208; à l'exception des dépôts de Giberville (Documentation inédite, V. Carpentier, Inrap) et Évreux II (BOMPAIRE 1984, p. 500-502). 


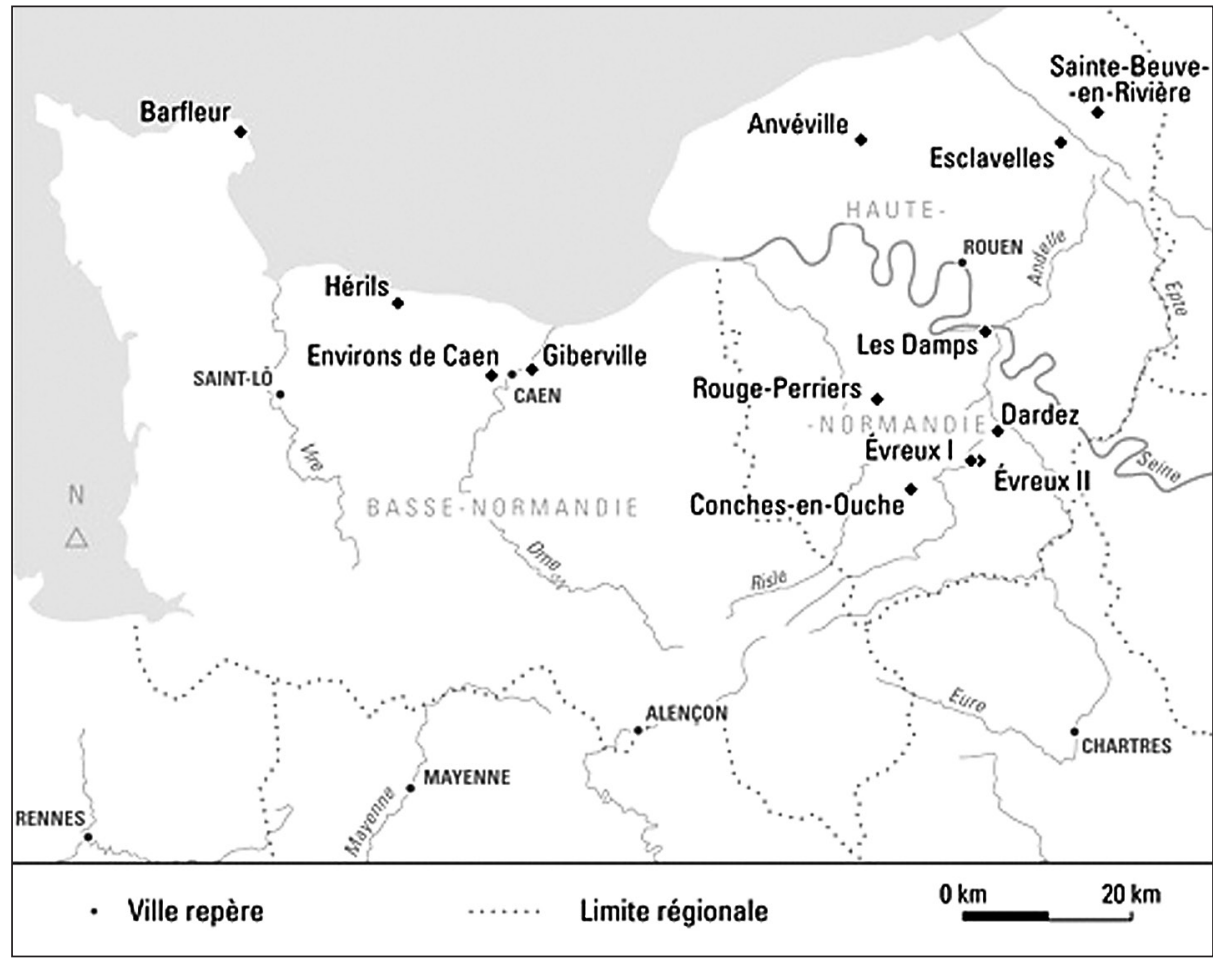

Figure 6 - Carte des trésors de bronzes mis au jour en Basse-Normandie se terminant par des émissions du IIIe siècle (DAO : Jean-Claude Fossey, UMR 6273, CRAHAM).

- Les Damps (Eure) - Découverte en 1785 ou 1786 dans trois vases en terre cuite de grands bronzes de Trajan, Hadrien, Sabine, Antonin le Pieux (en nombre), Marc Aurèle, Faustine, Aelius César, Commode, Crispine, Lucille, Pertinax, Septime Sévère, Julia Domna, Alexandre Sévère et Julia Mamaea.

- Dardez (Eure) - Découverte en 1874 de 166 grands bronzes de Nerva, Trajan, Hadrien, Sabine, Antonin le Pieux (30), Faustine mère et fille, Marc Aurèle (20), Lucius Verus, Lucille, Commode, Crispine, Dide Julien, Septime Sévère, Julia Domna, Alexandre Sévère, Julia Mamaea, Gordien III et Postume (4).

- Évreux I (Eure) - Découverte en 1894 dans un grand vase en terre grise de 662 grands bronzes, dont l'inventaire a révélé : 1 Auguste, 2 Vespasien, 10 Domitien, 8 Nerva, 63 Trajan, 132 Hadrien, 3 Sabine, 2 Aelius, 95 Antonin le Pieux, 34 Faustine, 80 Marc Aurèle, 53 Faustine jeune, 15 Lucius Verus, 31 Lucille, 61 Commode, 3 Crispine, 2 Albin, 7 Septime Sévère, 2 Caracalla, 1 Élagabale, 4 Alexandre Sévère, 1 Julia Mamaea, 2 Maximin I, 1 Gordien III et 49 incertaines. 
- Évreux II (Eure) - Découverte en 1983 de 294 bronzes (dont 11 indéterminés) répartis entre les périodes flaviennes (12), antonines (258), sévériennes (12) et post-sévériennes (1).

- Rouge-Perriers (Eure) - Découverte au XIXe siècle dans un vase en terre de 10 grands bronzes de Titus, Vespasien, Hadrien, Antonin le Pieux, Commode et Septime Sévère.

- Aux environs de Caen (Calvados) - Découverte en 1851 d'une quarantaine de grands bronzes dont 2 Commode et 7 ou 8 Postume.

- Hérils (Calvados) - Découverte en 1826 dans un vase en terre d'une centaine de grands bronzes depuis Domitien jusqu'à Alexandre Sévère.

- Giberville (Calvados) - En 2007, lors de la fouille d'un bâtiment du début $\mathrm{du} \mathrm{III}^{\mathrm{e}}$ siècle à vocation utilitaire, découverte de 4 sesterces, parmi lesquels 1 Antonin le Pieux et 3 Hadrien. Les monnaies étaient empilées au fond d'un pot, suggérant qu'un tissu les enveloppait.

- Barfleur (Manche) - Découverte en 1842 de 1500 grands bronzes depuis Galba jusqu'à Philippe père.

Remarquons d'emblée que le stock d'espèces en circulation privilégie le monnayage de bronze du IIe siècle, auquel s'ajoute un ensemble de monnaies issues du rer siècle. Il est vrai cependant que ce phénomène, partout perceptible et portant témoignage d'une difficulté de pénétration du bronze après 192, n'a pas connu le même développement dans toutes les régions (tableau 4). La Britannia est la seule où la prédominance du numéraire antonin apparait amortie par rapport à ce qu'on observe en Gaule. Cette observation, déjà soulignée par M. Amandry et D. Hollard dans leur étude du trésor de Bourg-Blanc (Finistère), s'expliquerait par le fait que la province insulaire se caractérise par un stock proportionnellement plus important de monnaies du Ier siècle ${ }^{48}(12,38 \%$ en moyenne). Sur le continent la situation est différente. Les monnaies du IIe siècle prédominent et marginalisent largement celles du Ier siècle ( $3,4 \%$ en moyenne). Or, les matrices utilisées pour la confection des moules de Sées s'inscrivent tout à fait dans ce contexte général (tableaux 2-3) et on lira sans peine dans ce fait son appartenance au stock d'espèces de bronze circulant en Gaule vers 250.

Une rapide lecture de la composition des trésors permet de mettre l'accent sur une autre particularité de la circulation du bronze en Gaule. Dans les dépôts enfouis au début du IIIe siècle, il est intéressant de constater que les sesterces sont toujours privilégiés, et ce très fortement ${ }^{49}$. À partir du milieu du mie siècle la tendance évolue. Les sesterces y sont peu nombreux et, surtout, cèdent le pas devant les as et les dupondii : c'est bien là le profil des moules de Sées.

48. Amandry, Hollard 2005-2006 (2007), p. 121-122.

49. Voir sur le sujet les commentaires de Buttrey 1972, p. 56 et Bastien 1978, p. 790-792. 


\section{Les raisons d'un surmoulage à Sées}

On conviendra que les moules de Sées ne sauraient alimenter un long discours sur l'importance du bronze dans les échanges de la cité sagienne. Au reste, quelle valeur avait-il alors pour susciter l'intérêt des monnayeurs locaux ? Une analyse fine des empreintes montrerait que ceux-ci ne firent pas n'importe quel choix. Le surmoulage a essentiellement porté sur des as et des dupondii à l'effigie radiée, et non sur des espèces laurées ${ }^{50}$. L'officine s'est donc montrée à la fois soigneuse et sélective dans le choix des types. Cela étonne et laisserait surtout supposer qu'il y avait un réel intérêt à surmouler des monnaies radiées plutôt que laurées. La raison en est sans doute à chercher dans la politique monétaire de Postume.

Peu après son accession au pouvoir, Postume entreprit de réformer le monnayage de bronze au cours du second semestre $260^{51}$. La réforme s'inspira de la politique de Trajan Dèce, puisqu'elle déboucha sur la création d'un double sesterce arborant une effigie impériale coiffée d'une couronne radiée ${ }^{52}$. Précisément, le but était de donner à la nouvelle monnaie une valeur supérieure à celle du sesterce traditionnel (et lauré). Pourtant, elle n'était qu'une émission de bronze d'un poids identique ou à peine plus lourd que les espèces laurées antérieures. La mesure était drastique : le même poids acquérait ainsi une majoration de $100 \%$, ce qui revenait à conférer au double sesterce de Postume une valeur libératoire plus élevée ${ }^{53}$. Si la réforme permit certainement d'élargir un stock en petit numéraire limité au bronze hérité du Haut-Empire ${ }^{54}$, elle se révéla être très vite un échec. En instaurant un change entre bronzes laurés et radiés qui ignorait leur proximité pondérale, il devenait difficile de faire circuler en même temps les deux monnaies. La réforme ne pouvait apparaître que défavorable aux espèces laurées et on imagine très bien qu'elle ait pu engendrer un phénomène de défiance. Pour s'en convaincre, il suffit de porter attention à la vague d'épargnes, associant sesterces du Haut-Empire et bronzes laurés de Postume, qui survint en $261^{55}$. Dans ce contexte, on s'expliquerait que la frappe régulière du bronze ait été suspendue dès le début du $3^{\mathrm{e}}$ consulat (262) de l'usurpateur gaulois ${ }^{56}$.

50. Parmi les moules de Sées, deux empreintes présentent néanmoins une tête laurée (tableau 2 : matrices $\mathrm{H} 3$ et $\mathrm{H} 7$ ), ce qui contredirait l'hypothèse selon laquelle la préférence pour les fractions radiées du sesterce a été délibérée. Toutefois, l'examen de ces empreintes indique qu'elles ont été réalisées à partir d'originaux déjà très usés. Il y a forte présomption pour que les faussaires ne se soient pas rendus compte qu'ils utilisaient des types laurés.

51. BASTIEN 1967, p. 49.

52. La nouvelle dénomination apparaît au cours de la deuxième émission de monnaies en bronze : cf. GRICOURT 1990, p. 47.

53. BASTIEN 1967, p. 30-31.

54. Cf. infra.

55. Voir les dépôts du groupe I (terminus post quem en 260-261) de Hollard 1992, p. 77-79. 56. Bastien 1967, p. 31. 
Toutefois, l'instauration d'une espèce de valeur supérieure eut pour conséquence d'ouvrir le champ aux monnayeurs locaux. Preuve, s'il en était, que l'effigie radiée de Postume jouissait d'une grande faveur, source de profits. Divers documents numismatiques l'attestent. C'est le cas notamment de la fabrication de faux doubles sesterces par coulage, que l'on retrouve en nombre dans les dépôts enfouis entre 268 et $270^{57}$. Autre fait notable : les surfrappes ou encore les contremarques qui, sans modifier l'effigie, remplacent par une couronne radiée les lauriers de la dynastie antonine ${ }^{58}$. Les monnayeurs de Sées ont-ils également cherché à profiter de la prime liée à la couronne radiée ? Ce n'est pas impossible et nous tiendrons pour déterminant le fait que le surmoulage ne porte, quasi exclusivement, que sur des émissions présentant une effigie impériale radiée. On en trouve une confirmation supplémentaire avec les matrices $\mathrm{H} 3$ et H4 (tableau 3). La présence d'un navire au revers évoque sans aucun doute le double sesterce de Postume à la légende LAETITIA AVG.

Une question reste encore posée : pourquoi les monnayeurs de Sées n'ont-ils pas alors surmoulé directement la monnaie de Postume ? Pour y répondre, la piste de recherche la plus commode à explorer est encore celle des trésors. Précisément, il apparaît que la région Nord et la Picardie constituent les zones d'élection du monnayage de bronze à l'effigie de Postume ${ }^{59}$. D'autres découvertes montrent qu'il a également pénétré dans l'actuelle Normandie, où il semble cependant n'avoir connu qu'une diffusion limitée au nord et à l'est de la région (figure 7). De fait, la cité sagienne se trouverait dans les marges de sa zone réelle de circulation. On doit en déduire que lorsque commença le surmoulage, la monnaie de Postume était certainement peu représentée dans les circuits monétaires. Dans ce contexte, les bronzes du Haut-Empire conservaient logiquement leur valeur et il y avait un intérêt majeur à recycler, par la pratique du surmoulage, ceux arborant un buste radié. Le bénéfice était d'autant plus assuré que les inventaires de trouvailles démontrent que le monnayage du Haut-Empire était thésaurisé et circulait en même temps que le monnayage de bronze de Postume.

La mise en évidence à Sées d'une production par surmoulage de monnaies en bronze nous renseigne donc sur la façon dont était reçu ce métal au tout début des années 260 dans une cité des confins septentrionaux de l'Empire. Réduit sans doute à ce qui subsistait de l'époque antonine, il y circulait toujours activement - pour une part malheureusement impossible à évaluer. Mais, en surmoulant des bronzes du Haut-Empire à l'effigie radiée, le phénomène gagnait encore en originalité. Il répondait à la réforme de 260 , qui installait alors la monnaie radiée comme maître du marché. C'était finalement le moyen d'utiliser, avec profit, un stock d'émissions anciennes, dans un système en cours d'évolution.

57. Gricourt, Hollard 1992, p. 26 et 48-49.

58. Naster 1951, p. 162, n. 19 ; Thirion 1960, p. 99-101 ; Bastien 1967, p. 30.

59. Hollard 1992, p. 89. 


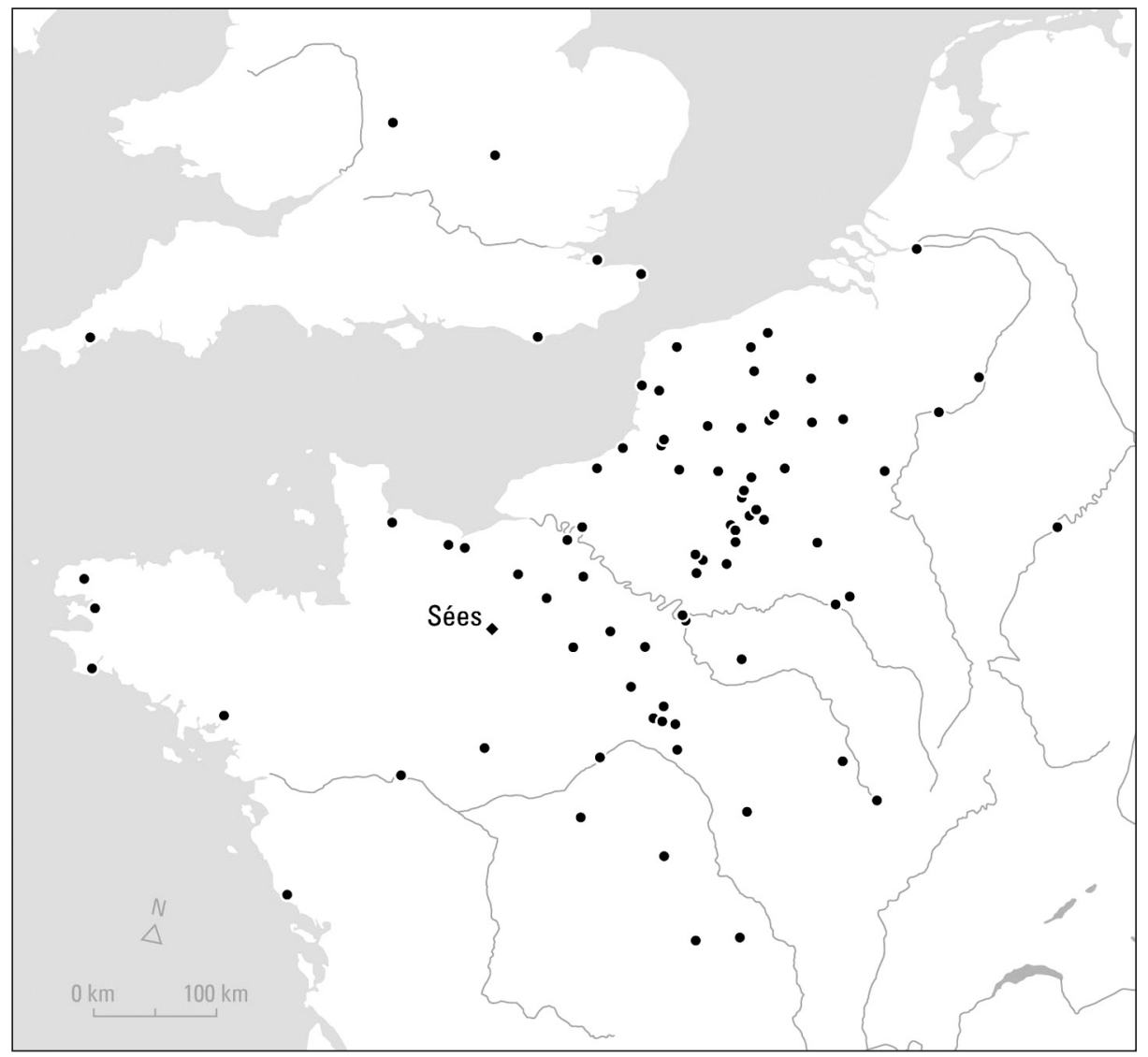

Figure 7 - Carte des découvertes de bronzes à l'effigie de Postume (d'après HOLLARD 1992).

\section{Conclusion}

Même réduits à quelques fragments, les moules de la rue Amesland et de l'église de l'Immaculée Conception à Sées constituent un document de grand intérêt. Témoins archéologiques de l'activité d'une officine installée au cœur de la cité sagienne, ils permettraient de dater la production de celle-ci du début de la décennie 260. Surmoulant des monnaies héritées du Haut-Empire, ils révèlent l'importance que revêtait, au cœur de la grande crise, la monnaie de bronze pour les petits échanges dans les confins septentrionaux de l'Empire. Faut-il alors y lire le reflet d'une mesure locale et tolérée, répondant à un besoin de la communauté ? Les textes n'évoquent pas le cas de la fausse monnaie de bronze. 
On serait donc enclin à considérer qu'elle ne constituait pas un délit à la différence notamment de la fausse monnaie d'argent. Toutefois, l'argument tiré du silence des textes n'a qu'une valeur relative : l'absence de dispositions réglementaires n'implique pas automatiquement que ces textes n'ont pas existé. Comme nous l'avons remarqué, la lex Cornelia de falsis (81 av. J.-C.), dans sa version actualisée au Haut-Empire, si elle ne se prononce pas sur le bronze, condamne en revanche la technique du coulage de fausses monnaies ${ }^{60}$. Dans ce contexte, l'hypothèse d'un atelier clandestin dont l'activité serait tolérée, voire encouragée par « un fonctionnaire local (militaire plutôt que civil) s'ingéniant à lutter contre une certaine pénurie de numéraire» selon les termes de J. Schwartz ${ }^{61}$, doit être repoussée. $\mathrm{Au}$ fond, elle signifierait surtout un laisser-faire incompréhensible doublé d'une perte de profit pour l'État - les monnaies de bronze étant des monnaies fiduciaires. Le phénomène identifié à Sées doit dès lors être avant tout considéré comme le résultat d'une production privée et éminemment frauduleuse, entreprise de petite ampleur à une époque où la structure du système monétaire dut favoriser une telle initiative. Parmi les types contrefaits, c'est bien le surmoulage d'espèces radiées qui permettait d'espérer le bénéfice le plus important. Selon nous, la production du faux-monnayeur prend en effet place à un moment (ca. 260) où le sesterce à la couronne radiée jouissait d'une valeur libératoire supérieure à celle du sesterce lauré. Cette configuration apparaît idéale et nul doute que le(s) faussaire(s) de Sées chercha, par choix ou par opportunité, à en tirer profit.

\section{Bibliographie des trésors présentés au tableau 4}

\section{Britannia}

Knapwell, Housesteads, Verulamium, Eastern Tower, Owston Ferry, Leysdown: A. Robertson, An inventory of Romano-British Coin Hoards, London, 2000, nos 361, $364,402,407,486$.

\section{Gaule}

Arnouville-lès-Gonesse (Val-d'Oise) : S. DE Turckheim-Pey, La trouvaille d'Arnouville-lès-Gonesse (68-238 après J.-C.), TM, III, 1981, p. 17-26.

Beveren-Leie (Belgique) : M. THIRION, Les trésors monétaires gaulois et romains trouvés en Belgique, Bruxelles, 1967, p. 53-54, no 27.

Bordeaux (Gironde) : Th.V. ButrRey, A Hoard of sestertii from Bordeaux and the problem of bronze circulation in the third century AD, ANSMN, 18, 1972, p. 33-58.

Bourg-Blanc (Finistère) : M. Amandry, D. Hollard, Le trésor de Bourg-Blanc 1989 (Finistère), TM, XXII, 2005-2006 (2007), p. 119-132.

60. Cf. note 25 .

61. Schwartz 1975, p. 208, suivi par Gricourt 1993, p. 564-566 ; Peter 2004 ; Pilon 2010. 
Bourges 7 (Cher) : P. BAstien, A. Cothenet, Trésors monétaires du Cher (Lignières : 294-310; Osmery : 294-313), Wetteren, 1974, p. 18, no 15.

Chilleurs-aux-Bois (Loiret) : Y. DE KISCH, Informations archéologiques, Circonscription archéologique du Centre, Gallia, XXXVI, 1978, p. 286-287.

Clémont-sur-Sauldre (Cher) : P. BAstien, A. Cothenet, Trésors monétaires du Cher (Lignières : 294-310 ; Osmery : 294-313), Wetteren, 1974, p. 20, no 26.

Epiais-Rhus (Val-d'Oise) : P.-H. MitARD, La trouvaille d'Épiais-Rhus (Val-d'Oise), TM, VII, 1985, p. 9-32.

Flobecq (Belgique) : M. ThiRION, Les trésors monétaires gaulois et romains trouvés en Belgique, Bruxelles, 1967, p. 79, no 91.

Froidmont (Belgique) : P. NASTER, Trouvaille de sesterces à Froidmont (1949), RBN, 97 , 1951, p. 161-166 ; M. ThiRion, Les trésors monétaires gaulois et romains trouvés en Belgique, Bruxelles, 1967, p. 84, no 102.

La Groutte (Cher) : P. BAstien, A. Cothenet, Trésors monétaires du Cher (Lignières : 294-310 ; Osmery : 294-313), Wetteren, 1974, p. 23, no 36.

Manage (Belgique) : M. THIRION, Les trésors monétaires gaulois et romains trouvés en Belgique, Bruxelles, 1967, p. 115, no 181.

Marboué «Annouillet» (Eure-et-Loir) : S. DE TuRCKheim-Pey, Le trésor de sesterces de Marboué («Anouillet»), Bull. Soc. dunoise, 275-276, 1986, p. 27-40.

Mélin (Belgique) : M. ThiRion, Les trésors monétaires gaulois et romains trouvés en Belgique, Bruxelles, 1967, p. 118-119, no 192.

Morialmé (Belgique) : M. ThiRION, Les trésors monétaires gaulois et romains trouvés en Belgique, Bruxelles, 1967, p. 126, no 210.

Primelles (Cher) : P. BAstien, A. Cothenet, Trésors monétaires du Cher (Lignières : 294-310 ; Osmery : 294-313), Wetteren, 1974, p. 25, no 48.

Puy-Dieu (Haute-Vienne) : J.-L. DesniER, Le trésor du Puy-Dieu, TM, VII, 1985, p. 33-104.

Sainte-Montaine (Cher) : P. BAstien, A. Cothenet, Trésors monétaires du Cher (Lignières : 294-310 ; Osmery : 294-313), Wetteren, 1974, p. 26, no 54.

Solre-Saint-Géry (Belgique) : M. THIRION, Les trésors monétaires gaulois et romains trouvés en Belgique, Bruxelles, 1967, p. 149, no 274.

Villaines (Sarthe) : D. Hollard, Le trésor de bronzes de Villaines-la-Carelle (Sarthe), TM, XXII, 2005-2006 (2007), p. 37-55.

\section{Bibliographie}

Abdy 2006 : R. Abdy, The decline and fall of the sestertius in Roman Britain: a case study, dans Asolati, Gorini 2006, p. 45-65.

AKerman 1838-1839 : J. AKerman, Roman coins mould, NC, 1838-1839, p. 147-161.

Amandry, Hollard 2005-2006 : M. Amandry, D. Hollard, Le trésor de Bourg-Blanc 1989 (Finistère), TM, 2005-2006 (2007), p. 119-132.

Andrieux et al. 2005 : Ph. Andrieux, Ph. Fluzin, V. Pichot, M. El-Amouri, O. Picard, Études expérimentales et métallurgiques du moulage de monnaies en bronze de la fin de l'Empire romain, dans L'exception égyptienne?, O. Picard, Fr. Duyrat (éd.), (Études alexandrines, 10), Paris, 2005, p. 225-252. 
AnOnyme 1890 : Anonyme, Exposition bibliographique de Sées (2-6 octobre 1889), Bulletin de la Société historique et archéologique de l'Orne, T. 9, 1890, p. 39.

Asolati, Gorini 2006 : M. Asolati, G. Gorini (éd.), I Ritrovamenti monetali e la legge di Gresham (Atti del III Congresso Internazionale di Numismatica e di Storia Monetaria, Padova, 28-29 ottobre 2005), Padova, 2006.

Auberson et al. 2004 : A.-F. Auberson, H.R. Derschka, S. Frey-Kupper, Faux contrefaçons - imitations. Actes du quatrième colloque international du Groupe suisse pour l'étude des trouvailles monétaires (Martigny 2002), (Études de numismatique et d'histoire monétaire 5), Lausanne, 2004.

Aubin 2003 : G. Aubin, Les moules monétaires en terre cuite du IIIe siècle : chronologie et géographie, $R N, 2003$, p. 125-162.

Aubin, Monin 1996 : G. Aubin, M. Monin, Les moules monétaires du IIIe siècle de la rue Sœur-Bouvier à Lyon (Rhône), BSFN, 6, 1996, p. 112-116.

BMC III : C.H.V. MAtTingly, Coins of the Roman Empire in the British Museum. Vol. III. Nerva to Hadrian, London, 1936 (éd. révisée 1976).

BMC IV : C.H.V. Mattingly, Coins of the Roman Empire in the British Museum. Vol. IV. Antoninus Pius to Commodus, London, 1968.

Bastien 1967 : P. Bastien, Le monnayage de bronze de Postume, Wetteren, 1967.

Bastien 1970 : P. Bastien, Trésor de 69 monnaies de bronze coulées du Haut-Empire romain, $B S F N$, janvier 1970, p. 475-477.

Bastien 1978 : P. Bastien, Trésors de la Gaule septentrionale. La circulation monétaire à la fin du IIIe et au début du Ive siècles, Revue du Nord, LX, 1978, p. 789-812.

Bernardelli 2006 : A. Bernardelli, La tesaurizzazione di moneta di bronzo in Italia nel III secolo d.c. e la legge di Gresham: alcune considerazioni, dans Asolati, GoRINI 2006, p. 69-101.

Bernouis 1999 : P. Bernouis, Carte archéologique de la Gaule. L'orne, 61, Paris, 1999.

Besombes 2004 : P.-A. Besombes, Le monnayage d'imitation de bronze de Claude $\mathrm{I}^{\mathrm{er}}$ : fraude et non nécessité, dans Auberson et al. 2004, p. 31-41.

Besombes 2008 : P.-A Besombes, Monnaies de l'Empire romain, IV. Trajan (98117 après J.-C.), Paris/Strasbourg, 2008.

Blanchet 1900 : A. Blanchet, Les Trésors de monnaies romaines et les invasions germaniques en Gaule, Paris, 1900.

Bompaire 1984 : M. Bompaire, Le trésor de sesterces d'Évreux, BSFN, juin 1984, p. $500-502$.

Boon 1965 : G.C. Boon, Light-weights and Limesfalsa, NC, 1965, p. 161-174.

Boon, Rath 1965 : G.C. Boon, P.A. RAth, Third-century countterfeiting at Whitchurch, Somerset, Arch. Journal, 122, 1965, p. 13-51.

Bost et al. 1992 : J.-P. Bost, M. CAmpo, D. Colls, V. Guerrero, F. Mayet, Le trésor de Cabrera III : sesterces de Domitien à Valérien, dans L'épave Cabrera III (Majorque), Talence/Paris, 1992.

Buttrey 1972 : Th. V. Buttrey, A hoard of sestertii from Bordeaux and the problem of bronze circulation in the third century AD, $M N, 18,1972$, p. 33-58.

CAllu 1969 : J.-P. CALlu, La politique monétaire des empereurs romains de 238 à 311 , Paris, 1969. 
CÉCILlon et al. 2011 : C. CÉCILlon, R. NiCOT, T. Silvino, Des faux-monnayeurs à Lyon/ Lugdunum, L'Archéologue, 115, 2011, p. 57-59.

Chameroy 2007 : J. Chameroy, Münzgussformen des 3. Jahrhunderts in den Sammlungen des RGZM, Jahrbuch des RGZM, 54, 2007, p. 533-572.

Chameroy, Guihard 2014 (à paraître) : J. Chameroy, P.-M. Guihard (avec la collaboration d'A. BOCQUET-LiÉNARD et de X. SAVARY, «L'officine de faux-monnayeurs de La Coulonche (Orne) : nummi coulés de la Tétrarchie en Occident », NC, 174, 2014, p. 153-191, pl. 20-25.

Chameroy, LAmbot 2011 : J. Chameroy, B. LAmbot, Les monnaies romaines du sanctuaire du Népellier à Nanteuil-sur-Aisne (Ardennes), Bull. Soc. Archéo. Champenoise, 104, 2011, p. 36-37.

Clausse 1994 : R. Clausse, Un secteur du quartier gallo-romain au plateau de Mageroux à Saint-Mard (Virton), dans Un quartier artisanal de l'agglomération galloromaine de Saint-Mard (Virton), (Études et Documents, Série Fouilles, 1), Namur, 1994, p. 127-140.

Delmaire 1989 : R. Delmaire, Largesses sacrées et res privata. L'aerarium impérial et son administration du rve au vie siècle, (Collection de l'École française de Rome, 121), Roma, 1989.

Depeyrot, Hollard 1987 : G. Depeyrot, D. Hollard, Pénurie d'argent-métal et crise monétaire au IIIe siècle après J.-C., Histoire et Mesure, II-1, 1987, p. 57-85.

Desnier 1985 : J.-L. Desnier, Le trésor du Puy-Dieu, TM, 1985, p. 33-104.

DRESCHER 1973 : H. DRESCHER, Beobachtungen und Versuche zur Herstellung römischer Münzgussformen, 20. Bericht der staatlichen Denkmalpflege im Saarland, 1973, p. 95-99.

Étienne, Rachet 1984 : R. Étienne, M. Rachet, Le trésor de Garonne. Essai sur la circulation monétaire en Aquitaine à la fin du règne d'Antonin le Pieux (159161), Bordeaux, 1984.

Fichet de Clairfontaine 1998 : F. Fichet de Clairfontaine, Sées, une ville provinciale de l'Empire romain, Bull. Soc. Hist. et Archéo. de l'Orne, 107 (3-4), 1998, p. 19-43.

Giardina 1973-1974 : A. Giardina, Sul problema della fraus monetae, Helikon, 13-14, 1973-1974, p. 184-190.

Gazdac, Alföldy-Gazdac 2001 : C. Gazdac, A. Alföldy-Gazdac, The roman law against counterfeiting between theory and practice: the case of roman Dacia, Acta Musei Napocensis, 38 (1), 2001, p. 137-154.

Gricourt 1990 : D. Gricourt, Les premières émissions de Postume à Trèves, TM, 1990, p. 31-54.

Gricourt 1993 : D. Gricourt, La trouvaille de deniers et d'antoniniens coulés, dans Les fouilles de sauvetage au centre hospitalier d'Arras, Bulletin de la Commission départementale d'histoire et d'archéologie du Pas-de-Calais, XIII-3, A. JACQUES, D. Gricourt, Ch. Hosdez, M. Tuffreau-Libre (éd.), 1993, p. 558-571.

Gricourt, Hollard 1992 : D. Gricourt, D. Hollard, Le trésor de bronzes romains de Méricourt-l'Abbé. Recherches sur les monnayages d'imitation tardifs de Postume, TM, 1992, p. 15-43. 
Grierson 1956 : Ph. Grierson, The Roman law of counterfeiting, dans Essays in Roman Coinage presented to H. Mattingly, R.A.G. Carson, C.H.V. Sutherland (eds.), Oxford, 1956, p. 240-261.

Hiver de Beauvoir 1837 : Hiver de BeAuvoir, Notice sur un atelier monétaire découvert à Damery (Marne) en 1830, RN, 1837, p. 171-180.

Hollard 1992 : D. Hollard, La thésaurisation du monnayage de bronze de Postume : structure et chronologie des dépôts monétaires, TM, 1992, p. 73-105.

Hollard 1996 : D. Hollard, La circulation monétaire en Gaule au mie siècle après J.-C., dans KING, WIGG 1996, p. 203-217.

Hollard, Foucray 1995 : D. Hollard, B. Foucray, Le trésor multiple de deniers et d'antoniniens de Cravent (Yvelines), TM, 1995, p. 71-114.

Hollard, Pilon 2005-2006 : D. Hollard, F. Pilon, Le trésor et les monnaies de site de l'Espace du Palais à Rouen (Seine-Maritime), TM, 2005-2006, p. 57-118.

KING 1996 : C. KING, Roman copies, dans KING, WIGG 1996, p. 237-258.

KING, WIGG 1996 : C. KING, D. WIGG (eds.), Coin finds and coin use in the Roman World (The Thirteenth Oxford Symposium on Coinage and Monetary History, 25.-27.3. 1993), Berlin, 1996 (SFMA, 10).

KRÄNZL 1996 : K. KRÄNZL, Versuch einer Rekonstruktion des Münzumlaufes des Prinzipats bis zum Ende der Antoninianprägung in Noricum, dans KING, WIGG 1996, p. 265-272.

Kunisz 1980 : A. Kunisz, La monnaie de nécessité dans les provinces rhénanes et danubiennes de l'Empire romain dans la première moitié du III siècle, dans Les dévaluations à Rome. Époque républicaine et impériale 2 (Gdansk, 19-21 octobre 1978), (Collection de l'École française de Rome, 37), Rome, 1980, p. 129-139.

LaLlemand 1994 : J. LaLlemand, Les moules monétaires de Saint-Mard (Virton, Belgique) et les moules de monnaies impériales romaines en Europe : essai de répertoire, dans Un quartier artisanal de l'agglomération gallo-romaine de Saint-Mard (Virton), (Études et Documents, Série Fouilles, 1), Namur, 1994, p. 141-177.

LORIOT, SCHEERS 1985 : X. LORIOT, S. SCHEERs, Corpus des Trésors monétaires antiques de la France IV, Paris, 1985.

MANDy 1992 : B. MANDy, Sées RD3, Bilan Scientifique (SRA Basse-Normandie), 1992, p. 55.

NASter 1951 : P. NASter, Trouvaille de sesterces à Froidmont (1949), RBN, 1951, p. 161-166.

Peter 2004 : M. Peter, Imitation und Fälschung in römischer Zeit, dans Auberson et al. 2004, p. 19-30.

Pfisterer 2007 : M. Pfisterer, Limesfalsa und Eisenmünzen - römisches Ersatzkleingeld am Donaulimes, dans Numismata Carnuntina. Forschungen und Material (FMRÖ, III.2), Wien, 2007, p. 648-736.

Pilon 1998 : F. Pilon, La fabrication de monnaies d'imitation frappées et coulées à Châteaubleau (Seine-et-Marne) au III siècle après J.-C., TM, 1998, p. 77-106.

Pilon 2004 : F. PILON, La fabrication de fausses monnaies coulées à Châteaubleau (Seine-et-Marne) au III s. après J.-C., dans La moneta fusa nel mondo antico. Quale alternativa alla coniazione? (Arezzo, 19-20 settembre 2003), F.M. VANNI, S. Bussi (ed.), Milano, 2004, p. 235-262. 
Pilon 2010 : F. Pilon, L'atelier monétaire de Châteaubleau (Seine-et-Marne) et les monnayages d'imitations de la seconde moitié du IIIe siècle après J.-C. dans les provinces occidentales de l'Empire romain, Thèse de doctorat, Université Paris 10,656 p.

R.-AlFöldi 1971 : M. R.-AlFöLDI, Die Gußformen und gegossenen «Fälschungen » kaiserzeitlicher Münzen, Chiron, 1, 1971, p. 351-363.

RIC II : H. Mattingly, E. A. Sydenham, The Roman Imperial Coinage, II. Vespasian to Hadrian, London, 1926 (réimpression 1972).

RIC III : H. Mattingly, E. A. Sydenham, The Roman Imperial Coinage, III. Antoninus Pius to Commodus, London, 1930.

Schwartz 1975 : J. Schwartz, Moules monétaires, dans Numismatique antique. Problèmes et méthodes. Actes du colloque, Nancy (27 septembre - 2 octobre 1971), (Annales de l'Est, Mémoire, 44), Nancy / Louvain, p. 207-209.

STRACK 1931 : P.L. STRACK, Untersuchungen zur römischen Reichsprägung des Zweiten Jahrhunderts, I, Die Reichsprägung zur Zeit des Traian, Stuttgart, 1931.

Thirion 1960 : M. Thirion, Les trésors d'Elverdinge et de Werken, RBN, 1960, p. 81-198.

TuRCAN 1963 : R. TuRCAN, Le trésor de Guelma, étude historique et monétaire, Paris, 1963.

TuRCAN 1982 : R. TuRCAN, Les moules monétaires du Verbe Incarné (Lyon), TM, 1982, p. 9-29.

VondROVEC 2003 : K. Vondrovec, Die antiken Fundmünzen von Ovilavis-Wels (FMRÖ, IV.1), Wien, 2003.

Wolters 1999 : R. Wolters, Nummi Signati. Untersuchungen zur römischen Münzprägung und Geldwirtschaft, (Vestigia, 49), München, 1999.

WоYтек 2010 : B. Wоутек, Die Reichsprägung des Kaisers Traianus (98-117), Wien, 2010. 